\title{
FRANCISCO NAYARI HACE UNA PETICIÓN AL OBISPO JUAN RUIZ COLMENERO (1649). ANÁLISIS Y TRADUCCIÓN DE UNA CARTA ESCRITA EN NÁHUATL POR UN HABLANTE DE CORA
}

\author{
FRANCISCO NAYARI MAKES A PETITION TO BISHOP \\ JUAN RUIZ COLMENERO (1649). ANALYSIS AND TRANSLATION \\ OF A LETTER WRITTEN IN NAHUATL BY A CORA SPEAKER
}

ÁLVARO J. TORRES NILA

ReSumEn. Se presenta el análisis, la paleografía y la traducción de una carta escrita en náhuatl por Francisco Nayari, el tonati o dirigente cora, fechada en 1649. Francisco Nayari le escribe al obispo de Guadalajara Juan Ruiz Colmenero con el fin de informar al prelado, que los coras se mantienen dentro de un modo de vida cristiano. La afirmación de Nayari pareciera derivar de alguna acusación contra los coras, en tanto que asevera que son fieles a la Corona española, y que son los tepehuanes, vecinos suyos, quienes no manifiestan un comportamiento cristiano. El documento es muy rico tanto por el momento histórico que revela como por el hecho de que el náhuatl de Francisco Nayari es su segunda lengua. Esto es notorio porque hay "errores" en el náhuatl del documento que son muy sistemáticos. Francisco Nayari finaliza su carta con una información que el obispo no puede subestimar: Francisco Nayari fue bautizado por Miguel Caldera, el capitán que negoció la paz entre los grupos chichimecas que estuvieron insurrectos por cerca de cincuenta años y el virrey de Nueva España. El obispo Ruiz Colmenero debe creer lo que Nayari dice.

PALABRAS ClavE: coras, náhuatl, petición, época colonial.

Summary. The following text is the analysis, transcription, and translation of a letter written in Nahuatl by Francisco Nayari, the Cora tonati or leader of the Cora people, dated in 1649. Francisco Nayari writes to Bishop Juan Ruiz Colmenero in order to inform him that the Cora people maintain themselves within a Christian lifestyle. Nayari's statement seems to respond to some accusation against the Coras, since he states that they are loyal to the Spanish crown, that it is the Tepehuanes, their neighbors, who do not exhibit Christian behavior. It is an insightful document because of the historical moment it reveals, and because the Nahuatl language written by Francisco Nayari is his second language. The "mistakes" the letter contains are very systematic. Francisco Nayari ends his letter including information that the bishop cannot overlook: Francisco Nayari was baptized by Miguel Caldera, the captain who negotiated peace between the Chichimec groups who rebelled for approximately fifty years, and the viceroy of New Spain. Bishop Ruiz Colmenero must believe what Nayari says.

KEYWORDS: Coras, Nahuatl, petition, colonial period. 


\section{Localización de la carta, descripción y estudios}

El Archivo Histórico del Arzobispado de Guadalajara (AHAG) resguarda numerosa documentación colonial procedente de las diferentes comunidades que lo conformaron. Si bien la gran parte de los documentos están escritos en español, hay algunos que fueron redactados en lengua náhuatl, principalmente durante el siglo xviı. Entre los textos escritos en náhuatl, se encuentra uno que llama la atención por sus características textuales y por su contenido. ${ }^{1}$ Como ha señalado Yáñez Rosales (2017: 237-238), el náhuatl en que está escrito refleja que quien lo escribió, tenía náhuatl como su segunda lengua, por el número de "errores" de carácter fonológico, principalmente, que la carta presenta. Estamos hablando de Francisco Nayari, el tonati, líder cora, quien se dirige al obispo de Guadalajara, Juan Ruiz Colmenero, ${ }^{2}$ para aclarar informaciones que se dicen sobre él y su pueblo.

Se trata de un breve expediente que consta de los textos escritos en náhuatl por Francisco Nayari y su traducción al español, ambos fechados en 1649. Los textos en náhuatl son cuatro fojas, de las cuales una está escrita sólo por el recto y tres por ambos lados. El texto en español consta de dos fojas escritas por ambos lados. ${ }^{3}$

La carta, al parecer fue conocida por el jesuita José de Ortega en el siglo xviII (1994 [1754]: 28). A fines del siglo xIX, Alberto Santoscoy hizo un estudio de dicha petición y sugirió que se trata de dos cartas, además presenta la paleografía de los textos en náhuatl y de la traducción al español de 1649 (1899: 1-6). Ricardo Medina García en su tesis doctoral comenta que son tres cartas las que escribe Nayari al obispo Colmenero (2016: 209212), además hace un análisis lingüístico sobre la escritura y la compara con otros textos en náhuatl del occidente novohispano; hizo su propia paleografía y traducción al inglés, así como la glosa de la foja 2 y 2 vuelta (2016: 363-368); Medina García es quien inicia la catalogación de los documentos en náhuatl del AHAG junto con el texto de Nayari. Rosa H. Yáñez, como lo he señalado arriba, analiza lingüísticamente la carta y su contexto junto con otros documentos en náhuatl del norte de la Nueva Galicia, hace su propia

${ }^{1}$ El AHAG deposita los textos en náhuatl coloniales encontrados en una sola caja llamada "Documentos en náhuatl" en la cual se puede observar la forma de escritura de las comunidades indígenas del occidente e incluso saber si pudieron tenerla como segunda lengua (Yáñez Rosales, 2017).

${ }^{2}$ Estuvo al mando de la Diócesis de Guadalajara desde 1646 hasta su muerte en 1663.

${ }^{3}$ El expediente también viene acompañado de otras tres fojas que contienen una paleografía con parte de los textos en náhuatl y español. A pesar de que no tiene una fecha escrita, se deduce que es de principios del siglo XX, ya que el tipo de escritura y el papel utilizados son totalmente diferentes a los textos de 1649, además de que la paleografía es dedicada al obispo de Guadalajara José de Jesús Ortiz (1902-1912) y su autor es Félix Montes de Oca. 
paleografía, traducción y glosa de puntos específicos para los fines de su investigación (2017: 248-257). Raquel Güereca en su tesis doctoral transcribe lo realizado por Santoscoy y explica las formas de comunicación de la sierra (2018: 20, 452, 509-515). Por mi parte, englobaré las cuatro fojas localizadas en el AHAG como una misma petición, en tanto que considero que no hay indicadores en la carta suficientemente contundentes para pensar que se trata de más de un texto. ${ }^{4}$

\section{La carta en la sierra del Gran Nayar}

Esta zona ha sido de difícil tránsito. El señorío cora mantuvo el control en gran parte de la sierra del Gran Nayar, a pesar de que la parte más oriental desde las postrimerías del siglo XVI ya pertenecía a la Nueva España. Concretamente San Luis Colotlán fue uno de los primeros pueblos de frontera que desde entonces propició una coexistencia de dos formas muy diferentes de organización social (Neurath, 2002: 49). Para Neurath, la regionalización de la sierra es una combinación de tres vertientes: "la ecológica, la reconstrucción etnohistórica y el análisis de la organización social y los ciclos festivos", que muestra una similitud histórica de las comunidades indígenas conviviendo en la zona, las cuales desde época prehispánica formaron parte de un corredor de pueblos agricultores que conectaba las antiguas civilizaciones mesoamericanas con los pueblos sedentarios del sureste de Estados Unidos. Se distribuyeron en el sur por el eje neovolcánico de los estados de Jalisco y Nayarit, al poniente con las zonas costeras nayaritas y sinaloenses, al oriente con los semidesiertos de la altiplanicie central y al norte de la sierra Madre Occidental, donde comienzan los cambios climáticos graduales para presentarnos a la zona taracahita (2002: 52-53). Éstas son las latitudes conocidas como el Gran Nayar en el que compaginan grupos étnicos como los coras, huicholes, nahuas y tepehuanes del sur hasta nuestros días.

No obstante, "la situación que prevaleció en la sierra de Nayarit durante la Colonia fue el resultado de los procesos de reacomodo sociocultural que se iniciaron con las conquistas de [Nuño de] Guzmán, y no concluyeron sino hasta finales del siglo Xvi" (Neurath, 2002: 68). Los conquistadores y evangelizadores sólo lograron establecerse en el siglo XVIII pues la población indígena de esta región y otras norteñas, se resistió a la colonización.

${ }^{4}$ La primera ocasión que presenté la carta de Nayari fue en el Taller de los Amigos de las Lenguas Yutoaztecas celebrado en Cholula, Puebla, en 2012. A raíz de eso, seguí con mi propia investigación haciendo la paleografía, traducción y glosa. Agradezco a Rosa H. Yáñez Rosales sus comentarios que retroalimentaron a este trabajo. 
Después de la represión desatada por el virrey Antonio de Mendoza para sofocar la Guerra del Miztón (1540-1542), algunos de los grupos insurrectos sobrevivientes se refugiaron en la sierra, entre barrancos y terrenos muy irregulares, (Dávila Garibi, 1957: 437; Yáñez Rosales, 2001: 83), incluso la sierra del Nayar albergó a "apóstatas de todos colores", personas pertenecientes a las diversas castas que huían con fines particulares (Péron, 1997: 53; Güereca, 2018: 249-275). Sólo en 1722 se logró la conquista militar por parte de los españoles, y quienes realizaron la labor de evangelización durante algunas décadas, hasta antes de su expulsión, fueron los jesuitas.

Si bien el náhuatl ya era una de las lenguas en gran parte del occidente mesoamericano en el siglo xvI, posteriormente se extendió como lengua de evangelización y de administración colonial (Yáñez Rosales, 2008). Gracias a que se desarrolló el náhuatl "de doctrina" y el "de escribanía" (Mentz, 2009), esta lengua se llevó a ejercer en lugares no nahuahablantes como la sierra del Nayar, y otras zonas donde no era la lengua originaria de la región.

En el caso del documento de Francisco Nayari que se analiza en este trabajo, es claro que para dirigirse a las autoridades españolas, en este caso el obispo Juan Ruiz Colmenero, Nayari utiliza el náhuatl y no el cora como presumiblemente era su lengua materna. ${ }^{5}$ Los franciscanos o algún otro clérigo podrían traducirlo al castellano y aclarar la postura y la situación de evangelización de los grupos coras a quienes Francisco Nayari lideraba.

Por la fecha en que la carta fue escrita, las autoridades novohispanas tenían presente los levantamientos indígenas sucedidos en el occidente y norte de la Nueva España, como la Guerra del Miztón (1540-1542), la Rebelión de Guainamota (1585) y la Rebelión de los Tepehuanes (1616-1618). Es claro que siempre estuvo latente el riesgo de que las misiones religiosas, que ejercían como punto de avanzada para la colonización, se vinieran abajo en cualquier momento. Bajo esta perspectiva, el obispo Colmenero inicia su visita pastoral en junio de 1648, sabe que la frontera con la sierra del Nayar "ha dejado de avanzar" y es "refugio para los fugitivos y apóstatas", por lo que decide "tomar en sus manos la conquista espiritual de la región", pero mientras es conciliador y visita pueblos de fácil acceso, no lo hace igual para con aquellos que viven en el interior de la sierra, de tal modo que cuando el prelado incursiona en la zona cora, los días 5 y 6 de mar zo de 1649, pide al indígena Diego Felipe, ${ }^{6}$ gobernador de Zayamota, que mande su men-

${ }^{5}$ Francisco Nayari era de Tzacaimuta, es decir, de La Mesa del Nayar. Por lo tanto, debió haber tenido cora meseño como su lengua materna. Esta variante del cora ha sido descrita por Vázquez (1994).

${ }^{6}$ Existe una breve relación de lo que hizo el obispo Juan Ruiz Colmenero en esa incursión, escrita por su secretario Francisco Delarrosa, allí comenta: "la desolación de los lugares de la dicha frontera y de algunos de la tierra adentro por los vecinos fugitivos indios, negros, mulatos, mestizos y libres y esclavos que por vivir con 
saje al líder cora: "le ordena ni más ni menos que expulse de sus refugios a los apóstatas que ha acogido" para entregárselos a su intermediario (Péron, 1997: 53-55).

Entonces no es extraño que para el 15 de mayo de ese mismo año, Francisco Nayari escriba su carta al obispo de Guadalajara en náhuatl. Además, sigue la estructura de los textos coloniales en español. Inicia con un saludo, se presenta y comenta la razón de la carta. Luego aclara cuáles son los pueblos que dirige (Guazamota, Ayotuxpan y Guaxicori), ${ }^{7}$ distingue que los tepehuanes son "los verdaderos malvados", no los coras, pidiendo una disposición ${ }^{8}$ para que se guarde en el pueblo de Francisco Nayari: Tzacaimuta $^{9}$ (véase la foja 2 de la paleografía y traducción). En seguida narra la llegada de un marqués anónimo a Chapota probablemente encontrándose con Huainori Nayar ${ }^{10}$ (véase la foja 3 de la paleografía y traducción), en tanto describe que incluso él prestó gente para

poco trabajo, con ningún apremio y con retiro de la verdadera religión se van a lo más retirado de la dicha provincia del Nayar sin que hasta ahora se haya puesto en ejecución remedio contra tanto daño, resolvió escribir al bárbaro infiel que al presente gobierna la dicha provincia, amonestándole eche de ella a los fieles fugitivos de cualquier estado y condición que sean, y no dé lugar a que en adelante se admitan otros, con apercibimiento de que se procederá al remedio que no alcanzare esta blandura con todo rigor", misma que está disponible en su versión paleográfica en Relaciones. Estudios de Historia y Sociedad, núm. 69, 1997.

${ }^{7}$ Aunque en época colonial en Guaxicori y Guazamota habitaron tepehuanes, probablemente también convivieron con grupos coras; para Efraín Rangel Guzmán y Jorge Luis Marín García (2014: 159) "los movimientos de población indígena en la región estuvieron a la orden del día", por lo que los asentamientos se convirtieron en "amalgama" de diferentes grupos indígenas, de tal manera que cuando Francisco Nayari manda la misiva a Colmenero, deja en claro que esas comunidades fueran reconocidas bajo su tutela para no ser confundidas con los sectores "malvados", véase el mapa 1.

8 Probablemente sea el "salvoconducto" que le entregó Miguel Caldera a Francisco Nayari "en el que certificaba haber sido recibido de paz" (Güereca, 2018: 287) y "se concretó... a decir en ella que su recomendado le había salido al encuentro" (Santoscoy, 1899: XXII).

${ }^{9}$ Conocido posteriormente como la Mesa del Tonati o Mesa del Nayar, en donde se encontraba "el recinto ceremonial de mayor importancia en la zona" del culto al sol entre los siglos XVI al XviII (Téllez, 2014: 19). Después de la muerte de Francisco Nayari (1616), el lugar resguardó su osamenta que "reunía a toda la nación cora y era un valioso instrumento de unidad, valioso sobre todo por estar en un medio propicio al aislamiento de cada población, ya que era consultado [como oráculo] también por los vecinos del oriente, los huicholes, y ciertos grupos de la costa" (Hers, 1977: 19). El escrito de Nayari probablemente fue realizado en este lugar, Medina García comenta (2016: 211): "he writes that his community is Tzacamota, an independent town in El Gran Nayar". Neurath comenta que en la sierra existió un señorío independiente durante la época colonial gobernado por un linaje de los "soles" o "Tonatis" (derivado del término náhuatl tonatiub), y su centro político se localizaba en la Mesa de Nayar siendo "en este lugar — también conocido como Tsakaimuta o Toakamuta — se rendía culto al dios niño Piltzintli, que era el sol, 'Nuestro Padre' Tayaupa o, simplemente, el dios del Nayar" (Neurath, 2002: 48).

${ }^{10}$ Hijo de Francisco Nayari. Según Alberto Santoscoy, Pedro Huainori pudo empezar a gobernar a partir de 1616 (1899: LII), y fue él quien realmente escribió la carta bajo una especie de interlocutor con la osamenta de su padre y heredero del culto en Tzacaimuta (1899: LXI). 
la construcción del convento en Guazamota y en Guaxicori, así como siguen "guardando" el de Ayotuxpan, ${ }^{11}$ volviendo a enfatizar que todos son coras (véase la vuelta de la foja 3 de la paleografía y traducción). Después describe que ha hablado con el gobernador don Diego Felipe para convocar a una reunión (véase la foja 4 de la paleografía y traducción). Por último, menciona que él fue bautizado ${ }^{12}$ nada menos que por Miguel Caldera (véase la vuelta de la foja 4 de la paleografía y traducción), el negociador de la Guerra Chichimeca (Powell, 1977).

Para Nayari, es importante que el obispo Colmenero reconozca que ellos no son los rebeldes, es claro al delimitar a su pueblo y comentar sobre la construcción de los conventos, además de que se ha entrevistado con Diego Felipe y que en el pasado tuvieron contacto con el capitán Caldera, incluso es muy categórico al insistir varias veces "¡así entérate señor obispo!".

\section{El nábuatl de Francisco Nayari}

Al leer la petición de Francisco Nayari, se observan varios rasgos que indican que su lengua materna no es el náhuatl, sino que lo utiliza como segunda lengua. ${ }^{13}$ Un caso es la frecuente sustitución de "e" por "i", por ejemplo:

\section{1. corami}

para indicar el plural de 'coras', término que se esperaría como corameh.

Asimismo, hay presencia de "i" en contextos que no corresponden a la presencia de esta vocal. Algunos ejemplos son:

\section{2. tazo pilitzin}

para decir 'queridos hijos', cuando se esperaría tlazohpiltzin.

${ }^{11}$ Nayari afirma que tales lugares son parte de los coras, para mediados del siglo xvII ya existían los conventos de Guazamota (1606), Ayotuxpan (1607) y Guaxicori (1621) (Santoscoy, 1899: LXXII), Rangel y Marín (2014: 159) comentan: "Debido a que la fundación de casas religiosas iba en aumento, las necesidades de mano de obra indígena para hacer producir las haciendas y mantener los conventos, se multiplicaban cada vez más", así el tonati pudo tener estrategias diplomáticas para mantener una cierta libertad en la sierra.

12 Probablemente en Juchipila (Santoscoy, 1899: XXI-XXII) o Tepic (Güereca, 2018: 164).

13 Yáñez Rosales (2017: 250-257) describe en qué parece consistir la interferencia del cora en el náhuatl de la carta de Francisco Nayari. 


\section{3. miyexuiti \\ para decir 'muchos años', mientras que se esperaría miec xiuitl. \\ 4. neguati ni cora \\ para decir, 'yo soy cora', en tanto que se esperaría nehuatl nicora.}

Este mismo rasgo se manifiesta en español:

\section{5. miqueli ca de la...}

para indicar 'Miguel Caldera'.

\section{Conclusiones}

La existencia de la carta de Francisco Nayari por un muy largo tiempo sólo fue conocida por Santoscoy, e incluso muchos estudiosos la han creído perdida hasta nuestros días. Las aportaciones que yo doy con mi análisis son dos. En primer lugar, ofrecer una nueva traducción al español de un documento tan importante para la historiografía y lingüística náhuatl del norte de la Nueva Galicia, no con el afán de demeritar lo realizado por Alberto Santoscoy u otros autores, por el contrario, es agregar más información a la ya existente. En segundo lugar, la importancia de señalar que en el texto sí aparece Miguel Caldera, y que al señalarlo, el líder cora mostró la importancia que tuvieron las negociaciones de paz de la Guerra Chichimeca durante la primera mitad del siglo XVII.

Con la carta de Nayari se pueden "escuchar" las voces de aquellos grupos que estaban refugiados en la sierra, que para la historiografía regional la mayoría de las veces han estado siempre "calladas", debido a que los análisis y la reconstrucción del pasado recaen en fuentes escritas por los españoles, con lo cual se crea un discurso donde sólo se aprecia una versión. Si bien, lo más probable es que para 1649, quien escribiera la carta fuera su hijo Pedro Huainori como tonati, no deja atrás la importancia que los grupos nayaritas tuvieron hacia la figura de Francisco Nayari como el centro neurálgico regional desde finales del siglo xvi hasta posterior a la conquista de la sierra en 1722. Incluso las mismas autoridades novohispanas al tratar de desestabilizar el sistema político y religioso indígena, decidieron llevar la osamenta de Nayari a la ciudad de México, donde fue quemada públicamente, rompiendo el culto más importante de los habitantes de la zona. Presentar la carta de Francisco Nayari es dar a conocer las voces de la comunidad cora de mediados del siglo XvII. 


\section{Paleografía, traducción con análisis morfológico y glosas}

Para todos aquellos que se han adentrado a traducir y analizar textos nahuas de la época colonial, sean principiantes o experimentados, han afrontado grandes retos: "conjugar un riguroso análisis lingüístico con un amplio conocimiento de la realidad social e institucional de los pueblos que elaboraron esta documentación es un requisito indispensable para lograr su cabal comprensión" (Herrera et al., 2007: 149). La interdisciplinariedad es parte esencial para desarrollar un trabajo de tal índole, de manera que si no se conoce el contexto histórico de un escrito en náhuatl, este último podría pasar desapercibido, asimismo de forma viceversa, de nada sirve tener a la mano un rico bagaje documental en náhuatl si no se le da su debido análisis lingüístico. Este trabajo pretende brindar ambas partes, la primera que se expuso letras más arriba, y la segunda que viene a continuación.

La presentación del texto está dividida por enunciados, correspondiendo el primero a la paleografía literal, el segundo a su análisis morfológico conservando lo más posible las formas originales del texto, ${ }^{14}$ el tercero a la glosa y el cuarto presenta la traducción libre de mi autoría.

${ }^{14}$ En un principio esta línea se había realizado completamente con la normalización del náhuatl "clásico" bajo algunos lineamientos que presentan otros trabajos de traducción, análisis y glosado (Peralta et al., 2004: 179-206; Herrera et al., 2007: 149-184), pero para respetar la variante del náhuatl en que fue escrito la carta de Nayari, se decidió presentarlo así. 


\section{CARTA EN NÁHUATL DE FRANCISCO NAYARI AL OBISPO JUAN RUIZ COLMENERO (1649)}

TRADUCCIÓN CON ANÁLISIS MORFOLÓGICO Y GLOSAS

Foja 1 recto

1. Amatzinti ytechi poui nomahuiz tlatzo

Ama-tzin-ti i-techi-pohui no-mahuiz-tla[t]zo[h]

papel-H-ABS 3sG.POS-POSP-leer Pos-honorable-amar

Este papel que ha de leer mi ama-

2. piltzi Señor vispo yhuano maguiz tlatzo

pil-tzi $<\mathrm{n}>15 \quad$ señor $<0>$ bispo ihuan $\quad<\mathrm{n}>0$-maguiz-tla[t]zo $<\mathrm{h}>$ -

hijo-H señor obispo y 1 1sG.Pos-honorable-amar

do señor obispo y el honorable y ama-

3. pilitzin Rei matouiyo Dios a mitz motla

pili-tzin rey, ma to-tecuyo Dios amitz ${ }^{16}$-mo-tla

hijo-H rey que 1PL.Pos-señor Dios 2PL.OBJ-REFL-amar

do rey, que nuestro señor Dios los ame

4. ço ca pieli miyexuiti yhuan miye to

zo-ca-pie-li mie[c] xihuitl ihuan mie[c] to-

AUX-guardar-APL mucho año y mucho día

y guarde muchos años y muchos dí-

5. na li a mitz tlaocoliz nemiliz ti amitzi

nali amitz-tlaocoli-z nemiliz-ti amitz-

2PL.OBJ-Socorrer-FUT vida-ABS 2PL.OBJ-

as, les socorra en vida [que a] ustedes les

6. macaz 1649.

maca-z 1649 .

dar-FuT 1649 .

dé, 1649 .

${ }^{15}$ El uso de [ ] se colocará para indicar las letras que sobran y el uso de $<>$ se hará para las letras que faltan de la paleografía.

${ }^{16}$ En náhuatl clásico sería "amech". 
Foja 2 recto

1. ma toteuiyo dios amitz mopieli señor vispo

Ma to-tecuyo Dios amitz-mo-pie-li- ${ }^{17}$ señor obispo

que 2PL.Pos-señor Dios 2PL.OBJ-REFL-guardar-APL-FuT señor obispo

Que nuestro señor Dios los guarde a ustedes, señor obispo

2. $y u^{\mathrm{a}}$ nomahuiz tazo pilitzin tlatuan Rei yhuan oce

ihuan no-mahuiz-tazo[h]-pil-tzin tla[h]toa-ni ${ }^{18}$ rey ihuan oc-ce-

y 1sG.Pos-honorable-amar-hijo-H hablar-A rey y todavía-

y mi bonrado amado bijo del rey y de-

3. quinti tlatoqui matoteui Dios a mitzinco tla

quin-tin tla[h]to-que[h] ma to-tecuyo Dios amitz-tzin-co tla $^{19}$

otro-PL $^{20}$ hablar-A.PL que 1PL.POS-señor Dios 2PL.OBJ-H-LOC ${ }^{21}$ a

más señores, que nuestro señor Dios, a ustedes apre-

4. ço ca pieli mi yexuiti

zoh-ca-pial mie[c] xiuiti

mar-AuX-guardar mucho año

ciados (señores), los guarde muchos años.

5. yhuan neguati no toca Don franco nayari

Ihuan neguati no-toca don Franc $<$ isc $>0$ Nayari

y 1SG 1sG.Pos-nombre don Francisco Nayari

Y mi nombre es Don Francisco Nayari,

6. to teuiyo dios ne hi momaquilia nochi no

to-tecuyo Dios nechi-mo-maqui-lia nochi no-

2PL.POS-Señor Dios 1SG.OBJ-REFL-dar-APL todo 1SG.POS-

nuestro señor Dios, me regala a todos mis

7. piligua ni pactica yhuan yoquix nc chihuali

pil-guan ni-pactica ${ }^{22}$ ihuan iuhqui ni-c-chihua-l[t]i

hijo-PL 1sG.SUJ-alegre y ADV 1SG.SUJ-OBJ-hacer-CAUS

bijos, estoy alegre y así lo hago

${ }^{17}$ Medina no coloca el futuro (2016: 363).

18 Medina traduce: "ruler" (2016: 363).

${ }^{19}$ La segunda línea que presenta el análisis morfológico en la mayoría de las ocasiones va a la par de la paleografía literal, es decir, que no se presentará la palabra completa si no se encuentra en la primera línea.

${ }^{20}$ Medina glosa: "other-pl" (2016: 363).

${ }^{21}$ Medina no coloca el locativo (2016: 363).

${ }^{22}$ Medina traduce como: "I am healthy" (2016: 364). 
8. matica

mati-ca.

saber-AUX

saber.

9. yhuan aquimatiz qui quenami niunica

Ihuan a[n]-qui-mati-z-queh quenami[h] ni-oncan

y 2PL-OBJ-Saber-FuT-PL ADV 1sG.SUJ-acá

Y quienes se enteren de esta manera, [sepan] que acá, yo

10. ni christiano [tachado] niunca quenami unixtlali

ni-christiano ni-oncan quenami[h] o-nech-tlali

1sG.su-cristiano 1sG.sU-acá ADV PERF-1SG.0BJ-sentar

soy cristiano, yo acá de esta forma me senté

11. Rei yhuan quenami uni chili hui marques tlatoani

rey ihuan quenami[h] 0-nich ${ }^{23}$-ilhuia marqués tlahtoa-ni

rey y ADV PERF-0Bj-decir marqués hablar-A

con el rey y así me dijo el señor marqués:

12. ipapa amo nimo ne los ynahuacate peuani unichiliu

ipampa a[h]mo ni-mo-nelo-z i-nahuac ${ }^{24}$ tepehuan-meh o-nech-ilhui

CONJ no 1sG-ReEL-mezclar-fUT 3sG.Pos-cerca tepehuan-PL PERF-1SG.0BJ-decir

para que no me alíe 25 a los tepehuanes, me dijo

13. tlatoan marques axca ni matitica niunica teuqui totlatoa ${ }^{\mathrm{n}}$

tlahtoa-ni marqués axcan ni-mati-tica ni-on-catqui o-qui-tlahtoa

hablar-A marqués hoy 1sG.SUJ-saber-PROG 1SG.SUJ-DIR-estar PERF-OBJ-hablar

el señor marqués: hoy yo estoy sabiendo, yo allá bablé

14. milaua ca amo [tachado] ni moneloa san noyoqui ne chicocolia

$\begin{array}{llllll}\text { melahuac } & \text { a[h]mo } & \text { ni-mo-neloa } & \text { zan } & \text { noyuhqui } & \text { nech-coco-lia } \\ \text { verdad } & \text { no } & \text { 1sG.Su-REFL-mezclar } & \text { sólo } & \text { ADV } & \text { 1sG.OBJ-odiar-APL }\end{array}$

con la verdad, yo no me alio, sólo por eso me odian

15. tepeuani milauaca tlaualiloco amo ni moneloa yna

tepehuan-meh melauac tlaueliloc a[h]mo ni-moneloa i-na tepehuan-PL verdad malo no 1sG.SuJ-mezclar 3sG.Pos-

los tepehuanes, son los verdaderos malvados, yo no me alio

23 En náhuatl "clásico" sería "nech".

${ }^{24}$ Medina traduce: "with" (2016: 365).

25 Decidí traducir neloa por "aliar" ya que al parecer la intención de quien fuera la autoridad novohispana es convencer al líder nayarita de no "aliarse" con los tepehuanes. 
16. uaca neguati ni cora mochi no piliguan quasamota huac nehuati ni-cora mochi no-pili-guan Guazamota cerca 1sG 1sG.suJ-cora todos 1sG.Pos-hijo-PL Guazamota con ellos, yo soy cora. Todos mis hijos, los de Guazamota,

17. corami yhuan ayotochipa no piliguan corami cora-meh ihuan Ayotuxpan-meh no-pili-guan cora-meh cora-PL y Ayotuxpan-PL 1sG.Pos-hijo-PL cora-PL [son] coras y los de Ayotuxpan [son] mis hijos coras

18. yhuan guaxcore no piligua corami yxquichitimatiz $\begin{array}{llllll}\text { ihuan } & \text { Guaxicori } & \text { no-pili-guan } & \text { cora-meh } & \text { Ixquich } & \text { ti-c-mati-z } \\ \text { y } & \text { Guaxicori } & \text { 1sG.Pos-hijo-PL } & \text { cora-PL } & \text { todo } & \text { 2sG.SUJ-0Bj-saber-fuT }\end{array}$ y los de Guaxicori[son] mis hijos coras. Todo tú lo sabrás.

19. Señor vispo yhuan Rei espania unica umpa mo poaz

Señor obispo ihuan Rey de España, oncan Ompa mo-pohua-z Señor obispo y Rey de España ADV ADV REFL-contar-FuT Señor obispo y rey de España, allá donde estás. Allá se contará

20. mo yxpa ypapa moyolo pachiuiz yhuan tinechitlaçotazo mo-ix-pan ${ }^{26}$ ipampa mo-yollo pachiui-z ihuan ti-nech-tlazo[h] Pos-cara-LOC CoNJ 2SG.SUJ-corazón tranquilizar-FuT y 2SG.SUJ-1SG.oBJ-amaren delante de ti, para que tu corazón se tranquilice y tú me ama-

21. taz miyequi tinechi tlazotaz axca ni mitz pouilia notlatoli tla- $\mathrm{z}$ miec ti-nechi-tlazo[h]ta-z axcan ni-mitz-poui-lia, no-tla[h]tol -FuT mucho 2SG.SUJ-1SG.0Bj-amar-Fut hoy 1SG.SUJ-2SG.OBJ-contar-APL 1SG.POS-palabra rás mucho, tú me amarás hoy que te cuente, mi palabra

22. ticaquiz timoyolaliz ${ }^{27}$ yhua ni mitz yolalia amo te no notlatla

$\begin{array}{lllll}\text { ti-c-caqui-z } & \text { ti-mo-yolali-z } & \text { ihuan } & \text { ni-mitz-yolalia } & \text { ahmo } \\ \text { 2sG.SUj-oBj-escuchar-FuT } & \text { 2sG.Su-REFL-contentar-FuT } & \text { y } & \text { 1sG.SUj-2SG.oBj-contentar } & \text { no }\end{array}$

23. tlen no-tlatlaque 1sG.POS-pe tú escucharás, tú te tranquilizarás, y yo te alegro, estoy sin pe-

${ }^{26}$ Medina traduce: "before" (2016: 366).

${ }^{27}$ El verbo yolalia aparece varias veces en este documento; sólo es registrado por Cortés y Zedeño (1765: 56, 71-72), con los significados de: "conciliar nuevos amigos, contentar, halagar". Opté por la utilización de algunos sinónimos en español para que la traducción no resulte repetitiva. 
24. coliqualini unica

colli; ni-cualli nican

cado 1sG.suj-bueno aquí

cado; estoy bien aqui. ${ }^{28}$

25. Señor vispo hueli ni mitz tlatlaotia ti nechi paleuiz nicqui ne

Señor $<0>$ bispo hueli ni-mitz-tlatlau[h]tia ti-nechi-palehui-z ni-c-ne-

señor obispo poder 1sG.su-2sG.0B-pedir 2sG.SuJ-1sG.0B--ayudar-fuT 1sG.SUJ-OBJ-que

Señor obispo en verdad te pido, que me ayudes, yo quiero

26. quinauatili yehuati upoliui temaca ya marques ypapa

qui nahuatilli yehuati o-polihui ti-c-maca-ya marqués ipampa

rer disposición 3SG PRET-perder 2SG.SU-0BJ-dar-IPFv marqués CONJ

una disposición que él perdió, tú se la dabas al marqués para que

27. mo piaz ypa alitepeti tzacamota no alitepeu

mo-piya-z ipan altepetl Tzacaimuta n-altepe-uh.

REFL-guardar-FUT POSP pueblo Tzacaimuta 1sG.SUj-pueblo-Pos

se guardara en el pueblo de Tzacaimuta, mi pueblo.

28. no piaz amati yni nic qui nequi

No-pia-z ${ }^{29}$ amatl inin ni-c-nequi

1SG.POS-guardar-FUT papel DEM 1SG.SUJ-oBs-querer

Yo conservaré ese papel que quiero.

Foja 2 vuelta

1. yhuan ni $\mathrm{m}<\mathrm{i}>$ tztetlanilia notlanauatili mo poa metzti

Ihuan ni-mitz-tetlani-lia no-tlanahuatil Mo-pohua meztli

y 1SG.SUJ-2SG.OBJ-pedir-APL 1SG.POS-ordenamiento REFL-contar mes

Y yo te pido mi ordenamiento. En la cuenta del mes

2. caz to [tachado- "na"] li tonali nemi mayo umochiua amati

caztolli tonalli nemi mayo o-mo-chihua amati

catorce día andar mayo PERF-REFL-hacer papel

es día quince que va de mayo se bizo la carta.

${ }^{28}$ Medina traduce: "I am good" (2016: 367).

${ }^{29}$ La primera consonante podría ser también una $m$-, es extraño que cambian a no-piaz cuando es un tipo de impersonal. También podría ser que se está hablando de un tubo para guardarlo, porque piyaztli - es un tipo de tubo, y parece que el uso del posesivo con piya indica un sustantivo, no un verbo, zserá el documento enrollado? 
Foja 3 recto

1. ma totecuyo Dios mitz mo pieliz Señor vispo no mahuiz Ma to-tecuyo Dios mitz-mo-piya-z señor obispo, no-mahuizque 1PL.POS-señor Dios 2SG.0B-REFL-guardar-fut señor obispo 1sG.Pos-honorableQue nuestro Dios te guarde señor obispo, mi honorable,

2. tlaço pilitzin señor vispo quena mi timo yeztica aço oc ueli tlazo[h]-pili-tzin señor obispo, ¿Quenami[h] ti-mo-ye[t]ztica? Aço oc huel amar-hijo-H señor obispo ADV 2SG.SUJ-REEL-estar ADV ADV poder amado bijito señor obispo, ¿Cómo estás? Acaso puedas estar conten-

3. achitzin Dios mitz mo maquilia mochica huiliz señor vis

achi-tzin Dios mitz-mo-maqu-lia mochi ca hueli-z Señor Obisbien-H Dios 2SG.OBJ-REFL-dar-APL mucho ADV poder-FuT señor obis tito, Dios te dé mucho. Ojalá señor Obis-

4. po ma Dios mitz mo pieli miyexuiti

po ma Dios mitz-mo-piya-lia

po que Dios 2SG.OBJ-REFL-guardar-APL mucho año

po, que Dios te guarde muchos años.

5. nica unican qui mo mahuiz tlatotzin motetlacoliliz

Nican oncan qui-mo-mahuiz-tla[h]tol-tzin mo-tetlacoliliz aquí allá OBJ-REFL-honorable-palabra-H 2SG.POS-orden Aquí, allá, se bonra tu palabra, tu disposición,

6. motequi paholiz unicaqui hueli cenca unipa

mo-tequipacholiz o-ni-c-caqui hueli cenca ni-pa

2sG.SUJ-preocupación PERF-1SG.SUJ-OBJ-escuchar bien mucho 1sG.SUJ-ale

tu preocupación, la escuché bien, mucho me alegra,

7. qui nimoyolali nitenamic mocxtzin yhuan

qui, ni-mo-yolalia ni-tennamiqui mo-icxi-tzin ihuan

grarse 1SG.SUJ-REEL-contentar 1SG.SUJ-besar 2SG.POS-pies-H y

me consuela. Yo beso tus pies y

8. momatzin yhuan mo corona uni tenamic ihua
mo-corona
o-ni-tennamiqui
2SG.POS-mano-H y 2SG.POS-corona PRET-1SG.SUJ-besar
tus manos y tu corona. Yo la besé 
9. ypapa mohuelitiliz señor vispo no pilitzin

ipampa mo-huelitiliz señor $<0>$ bispo no-pili-tzin

conj 2sG.Pos-poder señor obispo 1sG.Pos-hijo-H

por tu poder señor obispo, mi bijito.

10. axca mochi nitlaliz ypa amati ypapa necili temex

Axcan mochi ni-tlali-z ipan amati ipampa neci-lia ti-mitz

hoy todo 1sG.Su-sentarse-fut PoSP papel conj aparecer-APL 2SG.SUJ-2SG.0BJ

Hoy todo lo asentaré en el papel porque se muestra [que], tú te

11. tequi pahoa nopilitzin Señor vispo quenami uni

tequipachoa no-pili-tzin señor $<_{0}>$ bispo, quenami[h] o-ni-

preocuparse 1sG.SUJ-hijo-H señor obispo ADV PERF-1SG.SUJ-

preocupas, mi hijito, señor Obispo. De esta forma yo

12. quichiua ya cenca

qui-chihua ya cenca

oBs-hacer ADV mucho

ya bice mucho.

13. [tachado] uala no mahuiz tlaço pilitzin

O-hualla ${ }^{30}$ no-mahuiz-tlazo[h]-pili-tzin

PERP-venir 1SG.Pos-honorable-amar-hijo-H

Vino mi honorable, amado bijito

14. tlatuani marques oa cichapota oquinocha

tla[h]toa-ni marqués o-a[h]ci-c Chapota o-qui-notza

hablar-A marqués PERF-llegar-PRET Chapota PRET-OBB-llamar

el señor marqués, llegó a Chapota, lo convocó [a Huanori],

15. ni man uya ompa oaci oqui na micquycxtzin

niman o-ya ompa o-a[h]ci-c o-qui-namiqui i-icxi-tzin

ADV PERF-ir ADV PERF-llegar-PRET PERF-OBj-besar 3SG.Pos-pies-H

luego fue allá, y llegó, besó sus piececitos, siguen.

${ }^{30}$ Se agregó el proclítico de pretérito para mantener la concordancia gramatical con los verbos que 
16. yhuan y matzin yhuan y corona y toca uanor

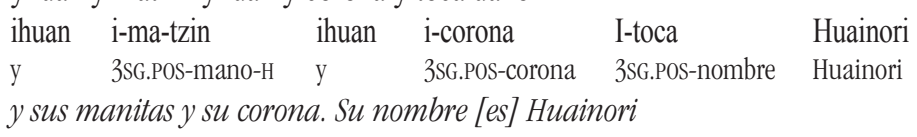

17. nayar [tachado] quali oqui napalo oqui mahuiztili yoqui umo

Nayar qualli o-qui-napalo o-qui-mahuizti-li iuhquin o-mo-

Nayar bien PERF-OB-gobernar PERF-0Bj-honrar-APL ADV PERF-REFL-

Nayar gobernó bien, se le respetó, así se bi-

18. chiua ypapa yhuelitiliz yoquix mati ${ }^{31}$ señor vispo

chihua ipampa i-huelitiliz iuhqui xi-c-mati señor obispo

hacer CoNj 3sG.Pos-poder ADV IMP-OBJ-saber señor obispo

zo debido a su poder jasí entérate señor obispo!,

19. yguac ca oqui maca tescati yhuan corona yhuan amati iguac ca o-qui-maca tezcati ihuan corona ihuan amatl CONJ ADV PERF-0BJ-dar espejo y corona y papel

cuando le dio el espejo y la corona y el papel.

20. ya nica mopia corona yhuan amati yaopoliu

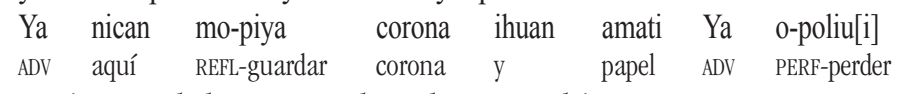

Aqui se guarda la corona y el papel. Ya se perdió

21. tez catini ca unica mopia Rei yaxca ynixmati

tezcati nican, oncan mo-piya rey i-axca in xi-c-mati espejo aquí ADV REFL-guardar rey 3SG.Pos-propiedad DEM IMP-OB-saber el espejo aqui; allá se guarda la propiedad del rey, entérate

22. señor obispo no pilitzin.

señor obispo no-pii-tzin.

señor obispo 1sG.Pos-hijo-H

señor obispo, mi bijito.

31 Considero que hay error de omisión de una letra, es decir, que se pretendía escribir xicmati "entiéndelo". En el documento aparece cuatro veces la frase yoquix mati señor obispo, que dentro del contexto de la carta lo interpreto como una oración imperativa "Así entiéndelo señor obispo". 
Foja 3v

1. señor vispo axca mochi ni mtz pohuliz no mahuiz tlacopilitzin

Señor $<0>$ bispo, axcan mochi ni-m<i>tz-pohu-ili-z,

señor obispo hoy todo 1SG.SUJ-2SG.0BJ-contar-APL-FUT

no-mahuiz-tlazo[h]-pili-tzin

1sG.Pos-honorable-amar-hijo-H

Señor obispo, hoy todo yo te narraré, mi honorable querido bijito.

2. yaui ca quenami omohiua umpa quasamota cano

ya huica quenami[h] o-mo-chihua ompa Guazamota ca no-

ADV ir ADV PERF-REFL-hacer ADV Guazamota ADV 1sG.POS-

[La cristianización] ya avanza de esta forma, se bizo allá en Guazamota

3. techicopa umochiua gobento omoualica totlatzin

techi-copa o-mo-chihua convento o-mo-hualica to-ta[h]-tzin

POSP-POSP PERF-REFL-hacer convento PERF-REFL-venir 1PL.POS-padre-H

gracias a mí, se hizo convento, nuestro padre vino de

4. guariana y toca frica fila totlatzin oaci quasamotla

Guadiana, i-toca Francisco Fila ${ }^{32}$ to-ta[h]-tzin, o-a $[\mathrm{h}] \mathrm{ci}-\mathrm{c}$

Guadiana 3SG.Pos-nombre Francisco Fila 1PL.Pos-padre-H PERF-llegar-PRET

Guazamota,

Guazamota

Guadiana, se llama Francisco Fila, llegó a Guazamota,

5. no techicopa umochihua yhua no piliguan uni

no-techi-copa o-mo-chihua ihuan no-pili-guan. 0-ni-

1SG.POS-POSP-POSP PERF-REFL-hacer y 1SG.POS-hijo-PL PERF-1SG.SUJ-

gracias a mi y a mis hijos se bizo [el convento]. Yo

6. nicmaca yaquitequi panoa Rei ycali yhua

ni-c-maca ya qui-tequipanoa rey i-calli ihuan

1SG.SUJ-0BJ-dar ADV OBs-trabajar rey 3SG.POS y

proporcioné [personas] que trabajan para el rey en su casa y

32 Aunque del documento en náhuatl no se desprende que el nombre corresponda a Francisco Fila, anoto aquí tal transcripción con base en el documento en español. 
7. tepixqueme yaqui paleuia no piliguan yaquali tepixque-meh $^{33}$ ya qui-palehuia no-pili-guan Ya cualli guardia-PL ADV OB-ayudar 1sG.Pos-hijo-PL ADV bien [proporcioné a] los vigilantes que ayudan a mis bijos. Así bien

8. uniquichiua yhua corami.

$\begin{array}{lll}\text { o-ni-qui-chihua } & \text { ihuan } & \text { cora-meh } \\ \text { PERF-1sG.SuJ-obj-hacer } & \text { y } & \text { cora-PL } \\ \text { bice, y los coras } & & \end{array}$

9. yhuan ayotohipa mopia gobento ca no piligua

$\begin{array}{lllll}\text { ihuan Ayotuxpan-meh } & \text { mo-piya } & \text { convento, ca no-pili-guan } \\ \text { y } & \text { Ayotuxpan-PL } & \text { REFL-guardar } & \text { convento ADV } & \text { 1sG.POs-hijo-PL } \\ \text { y los de Ayotuxpa [que] guardan el convento, son mis hijos. }\end{array}$

10. ca mochi nopiliguan yaquitequi panoa Rei ycali

\begin{tabular}{|c|c|c|c|}
\hline mochi & no-pil-huan & ya & qui-tequipanoa \\
\hline todo & 1sG.Pos-hijo-PL & $\mathrm{ADV}$ & oBJ-trabajar \\
\hline
\end{tabular}

Todos mis hijos ya trabajan la casa del rey.

11. ihuan tepixqueme ayototzipa corami.

Ihuan tepixque-meh, Ayotuxpan, cora-meh.

y guardia-PL Ayotuxpan cora-PL

Y los vigilantes, $y$ los de Ayotuxpan son coras.

12. yhuan yauiqui umochiua gobento guaxcore

ihuan o-ya-queh o-mo-chihua convento Guaxicori

y PERF-ir-PL PERF-REFL-hacer convento Guaxicori

Y [también] fueron para que se biciera el convento de Guaxicori.

33 Puede ser una variación de Tlapixqui que Alonso de Molina (1571: 132) registra como "el que guarda algo/ la persona que guarda". Diego Durán comenta que Tepixque "quiere decir guardas de gente" (1579, t. II: 223). Teresa Rojas Rabiela (1979: 49) comenta que Tepixque literalmente significa "Vigilante de gente", por lo menos para el centro de México, los Tepixques eran aquellos que vigilaban la ejecución del trabajo encomendado "era un nombre genérico para cuadrilleros, fueran de 1 o de 5 veintenas. Los tepixque debían escogerse entre los más conocedores (los tlamatinimi) de las mismas veintenas que estaban integradas al parecer sólo por maceguales, los de 20 familias. Se especifica que los canteros y carpinteros quedaban también integrados en las veintenas", este sistema se realizaba en el siglo Xvi y es probable que también en época prehispánica. De tal modo que Francisco Nayari, además de prestar mano de obra indígena, escogió a algunos coras para que fungieran como guardias/centinelas y estuvieran vigilando la construcción de los conventos que cita en su carta. 
13. mochi no piligua yaquite qui panoa Rei ycali

Mochi no-pil-huan ya qui-tequipanoa rey i-calli

todo 1sG.Pos-hijo-PL ADV oBs-trabajar rey 3SG.Pos-casa

Todos mis hijos ya trabajan la casa del rey.

14. yhuan tepixqueme yoqui nite maca

Ihuan tepixque-meh, iuhqui, ni-te-maca

y guardia-PL ADV 1SG.SUJ-0Bj-dar

Y los vigilantes, de esta forma, yo los doy,

15. no piliguan ynixqui mati señor vispo

no-pili-guan in xi-c-mati señor $<_{0}>$ bispo,

1SG.POS-hijo-PL DEM IMP-0B-saber señor obispo

son mis hijos, jentérate señor obispo!,

16. ypapa moyolo pahiuz ${ }^{34}$ yhuan guaxcore corami.

ipampa mo-yolo-pachihu-z Ihuan guaxicore-meh cora-meh

conj 2sG.Su-corazón-satisfacer-FuT y Guaxicori-PL cora-PL

para que tu corazón se tranquilice. Y los de Guaxicori son coras.

17. san yz quichi notlatoli [tachado] ticaquiz neguati

zan ixquich no-tla[h]toli, ti-c-caqui-z, neguati

ADV todo 1sG.POS-palabra 2sG.SUJ-OBJ-escuchar-FuT 1SG

Esta es toda mi palabra, tú la escucharás, yo soy

18. [tachado] to franco nayar

Francisco Nayari

Francisco Nayari

Francisco Nayari.

Foja 4 recto

1. señor vispo axca tic nequi nopiliguan yauiquetlati

Señor $<0>$ bispo axcan ti-c-nequi no-pili-guan yaui-qui [ilegible $]^{35}$ señor obispo hoy 2sG.SUJ-OBJ-querer 1sG.Pos-hijos-PL ir-0BJ

Señor obispo, boy tú quieres que mis hijos vayan a [i:?]

${ }^{34}$ En algunos documentos en náhuatl de época colonial, en especial los que yo he revisado del occidente de México, he visto que la "h" a veces es utilizada por el sonido de "ch".

35 No es clara la palabra. Pareciera ser tlatiya, que sería "escondía", o "quemaba", lo cual no corresponde al discurso de Francisco Nayari. 
2. ya uni celite tiquitoa amo mextequi pahos ni

$\begin{array}{llllll}\text { [ilegible] } & \text { o-ni-celi } & \text { tetequitia } & \text { a[h]mo } & \text { mitz-tequipachoa } & \text { ni- } \\ & \text { PERF-1sG.su-solo } & \text { repartir } & \text { no } & \text { 2sG.oBj-angustiar } & \text { 1sG.suj- }\end{array}$

Yo sólo repartí [el trabajo], no te angusties, yo

3. temacaz tlaco tlaco mocahuas ypapa noyo

te-maca-z tlaco, tlaco mo-cahua-z ipampa no-yuhqui

OBJ-dar-FUT mitad mitad REFL-dejar-fUT CONJ también-ADV

daré la mitad [de la gente], [la] mitad se dejará para mí, también así

4. qui no techimonequi quichiuaz qui nomilli yhuan

no-techi monequi qui-chihua-z-queh no-milli ihuan

1SG.POS-POSP necesitar 0Bj-hacer-FuT-PL 1SG.POS-sementera y

es necesario que bagan mi sementera y

5. no cali yoquix mati señor vispo

no-cali iuhqui xi-c-mati, $\quad$ señor $<0>$ bispo

1sG.Pos-casa ADV IMP-OB-saber señor obispo

mi casa. jAsí entérate Señor Obispo!.

6. ya uniquilliui Don Diego filibe governador

ya o-ni-qu-ilhui-li don Diego Felipe gobernador

ADV PERF-1SG.SUJ-OBJ-decir-APL don Diego Felipe gobernador

Ya le dije al gobernador don Diego Felipe

7. ypapa qui no notzas qui mo uitlauiz quitaliz

ipampa qui-nonotza-z qui-mo-cuitlahui-z qui-tlali-z

CONJ OBj-llamar-FUT OBJ--REFL-convocar-FUT OBj-reunirse-FUT

para que llame, que convoque a reunirse,

8. yoquix mati señor vspo yxquichi notlatoli

iuhqui xi-c-mati señor $<0>$ bispo Ixquich no-tla[h]tol

ADV IMP-OBB-saber señor obispo todo 1sG.POS-palabra

¡Así entérate señor obispo!. Hasta aquí toda mi palabra,

9. ticaquiz timo yolaliz timo papaquilltiz ni $\mathrm{mtz}$

$\begin{array}{lll}\text { ti-c-caqui-z } & \text { ti-mo-yolali-z } & \text { ti-mo-papahqui-lti-z } \\ \text { 2sG.suj-oBj-escuchar-FuT } & \text { 2sG.su-2sG.Pos-alegrar-FuT } & \text { 2sG.su-2sG.Pos-alegre-CAUs-FuT }\end{array}$

ni-m $<\mathrm{i}>\mathrm{tz}-$

1sG.SUJ-2SG.OBJ-

tú la escucharás, tú te sosegarás, estarás feliz, yo te 
10. yolalia neguati no toca Don fran ${ }^{\mathrm{co}}$ nayar yolalia nehuati. No-toca don Francisco Nayari contentar 1SG 1SG.Pos-nombre don francisco nayari daré serenidad. Me llamo don Francisco Nayari

11. yxquichi notlatoli. yxquichi no-tla[h]toli. todo 1sG.Pos-palabra hasta aquí toda mi palabra.

12. Señor vispo hueli ti nechi tlaçotlaz [tachado] ya ti caqui Señor $\quad<0>$ bispo hueli ti-nechi-tlazo[h]tla-z, ya ti-c-caqui Señor obispo ADV 2SG.SUJ-2SG.oBj-amar-FUT ADV 2SG.SUJ-oBj-escuchar Señor Obispo mucho tú me amarás, ya tú escuchas

13. notatoli teniquitoa ti nextlaço camatiz no-tla[h]toli ten-i[h]toa ti-nech-tlazo[h]camati-z 1sG.POS-palabra labio-hablar 2sG.suJ-1sG.0Bi-agradecer-FuT mi palabra, lo afirmo, tú me agradecerás.

14. yhuan no mahuiz tlazo pilitzin tlatuani Ihuan no-mahuiz-tlazo[h]-pili-tzin tla[h]toa-ni y 1sG.Pos-honorable-amar-hijo-H hablar-A Y mi bonorable amado bijito el señor

15. Rei ma nez tlaçutaca yca mochiyolo quena rey ma ne-z tlazo[h]-tlaca ica mochi yolotl quenami[h] rey que aparecer-fuT amar-persona INSTR todo corazón ADV rey, que aparezca el amado hombre, con todo el corazón, [asi] como

16. $\mathrm{mi}^{\mathrm{ni}}{ }^{\mathrm{q}}$ qi tlazotla maneli hueca unica espana ni-c-tlazo[h]tla, manel hue[h]ca, oncan España 1sG.SUJ-OBJ-amar coN lejos ADV España yo lo amo, aunque esté lejos, allá en España

17. niqui tazota yca mochi noyolo yxquichi notla ni-c-tazo[h]ta ica mochi no-yolo ixquich no-tla[h] 1SG.SUJ-0BJ-amar INSTR todo 1SG.POS-corazón todo 1sG.PoS-pa yo lo amo con todo mi corazón. Es toda mi pa- 
18. toli [tachado] ticaquiz no mahuiz tlatotzin
tol ti-c-caqui-z no-mahuiz-tla[h]to-tzin
labra 2SG.SUJ-oBs-escuchar-fuT 1sG.Pos-honorable-palabra-H
labra, tú escucharás mi honorable palabra.

Foja 4 verso

1. axca ni mitz matzilitiz notlatoli nicauala [tachado]

Axcan ni-mitz-mach-ilti-z no-tla[h]tol nican hualla

hoy 1sG.Su-2SG.0B-saber-caus-rut 1sG.pos-palabra aquí venir

Hoy yo te daré a conocer mi palabra: aqui vino

2. [capitana- tachado] miqueli ca de la yquaca

Miguel Caldera. I[h]cuac

Miguel Caldera coNj

Miguel Caldera. Cuando

3. [tachado] nechiqua tequi nel tucayoti Don franco na nech-quatequi nech-tocayoti don Francisco Na

1sG.obj-bautizar 1sG.obj-nombre don Francisco Na

me bautiza, [me otorga] mi nombre en la fe, don Francisco $\mathrm{Na}$

4. yari yhuan oce pauala partolome Soares

yari. Ihuan occe-pa hualla Bartolomé Suárez

yari y otra-vez venir Bartolomé Suárez

yari. Y en otra ocasión vino Bartolomé Suárez,

5. capitana yhuan oqui qua tequi nopilitzin

capitán ihuan o-qui-quatequi no-pil-tzin

capitán y PERF-0BJ-bautizar 1sG.POs-hijo-H

capitán, y bautizó a mi bijito

6. oqui toca yoti Do miqueli ceronimo ytlatzin

o-qui-tocayoti don Miguel Jerónimo i-ta[h]-tzin

PERF-OBJ-nombrar don Miguel Jerónimo 3sG.POS-padre-H

lo nombró don Miguel Jerónimo, su padrino

7. umochiua yoquix mati señor vispo yxqui

o-mo-chihua iuhqui xi-c-mati señor $<_{0}>$ bispo. Ixquich PERF-REFL-hacer ADV IMP-OB-saber señor obispo todo se bizo. jAsí entérate señor obispo! Es toda 
8. chi no tlatoli ni mo firma tia Don franco nayr. no-tla[h]toli ni-mo-firma-tia don Francisco Nayari.

1sG.POS-palabra 1SG.SU-REFL-firma-CAus don Francisco Nayari mi palabra, yo firmo don Francisco Nayari. 
CARTA DE FRANCISCO NAYARI DE 1649

PALEOGRAFÍA DE LA TRADUCCIÓN AL ESPAÑOL

Foja 1 recto

1. Carta escrita en lengua Mexicana, al Y[lus] ttt[risi]mo S[eño]r D[octo]r

2. D[on] Joan Ruis, Colmenero, [tachado] Dignissimo ob[is]po de la

3. S[an $]^{\text {ta }}$ Yglesia Cathedral de Guadalajara, Por D[on] Fran[cis $]^{\text {co }}$

4. Nayari, Yndio $=$ traducido como suena $=$

5. Este papel a de leer El famoso guarda de la Casa de Dios El

6. S[eño]r Ob[is]po Y tambien El famoso hijo del Rei, a quienes Dios

7. n[uest]ro S[eño]r g[uard]e y de muchos dias de vida en que se gozen. año de 1649

8. S[eño]r Ob[is]po Dios n[uest]ro s[eño]r te g[uard]e y a n[uest]ro famoso hijo del Rei y

9. tambien a los demas $\mathrm{S}[$ eño] res y ministros aquienes Dios g [uard]e muchos

10. años=

11. Y Yo que me llamo D[on] Fran[cis ${ }^{\text {co }}$ Nayari digo q[u]e a sido Dios servido

12. de darme salud, y a todos mis subditos $\mathrm{q}[\mathrm{u}] \mathrm{e}$ se hallan con ella $=$

13. Y tambien as de Saber Como estoi en el estado de Christiano

14. Conforme me puso El Rei, y Como me lo dijo aquel S[eño]r Marques

15. para que no me revolviese Con los Tepeguanes=

16. S[eño]r Marques eSabido que sedice que los mios los Comunican

17. La verdad es que tal no pasa ni comunico esa g[en]te, sino que es

18. tandome quieto ellos me andan abuscar q[u]e de verdad son

19. malos los Tepeguanes, y yo soi de los Coras, y los demas mis

20. subditos, Los Guasamotas, Coras, Ayotuspas, y Guaxicoras, estan

21. quietos y assi quiero que lo sepas=

22. S[eño]r Ob[is]po Y tambien El Rei q[u]e esta en españa, Lease este papel

23. en v[uest]ra Presencia, para que v[uest]ro Corason se quiete, y me querais 
24. mucho Como Yo os quiero, y ahora os digo lo que siento $\mathrm{p}$ [ar] a que

25. Io sepais y os holgueis y holgarme yo de que no tengo pecado

26. sino que estoi como me aveis puesto $=$

27. $S[$ eño]r $\mathrm{Ob}[$ is $]$ po mucho y Consumision te pido que nos ayudes, en $\mathrm{q}[\mathrm{u}] \mathrm{e}$

28. senos embie orden de lo quedevemos hazer $\mathrm{p}[\mathrm{ar}] \mathrm{a}$ que se guarde

29. en el Pueblo, porque la que nos dio El Marques senos a per

30. dido y deseamos tenedla y este es $n$ [uest] ro yntento $=$

31. Ytambien te digo que Ymbio este papel y Razon q[uan]do se cuentan

32. quince del mes de Mayo=

Foja 1 vuelta

Otro

1. S[eño]r Ob[is]po Dios te g[uard]e dime Como estas y si n[uest]ro P[adr]e Dios te

2. da Salud, este la Conserve y g[uard]e muchos años=

3. Los que aqui estamos tepedimos recibas La [e]n[h]orabuena de

4. que eSabido tus recaudos en que anparas n[uest] ros desconsuelos

5. y me huelgo mucho, y te beso los pies, las manos y tambien

6. tu Corona La Bese, porque sea alientan mis hijos=

7. Ahora pondre en este papel y Contare Como se desconsuela

8. mi hijo como yo lo hise otra vez=

9. Vino El famoso mi hijo El Marques y llego a chapota

10. llamo y luego se fue alla vesaronle Los pies y Las manos

11. y La Corona, llamase Vanorinaya Cargaronle bien y le

12. obedesieron Como mejor fuersa tuvieron, supolo El S[eño]r Ob[is]po

13. y que dejo un poco deseda, y Corona, y un papel y aqui

14. se guarda la Corona y el papel se perdio, guardamos La

15. orden del Rei y aora se esto y te lo digo S[eño]r $\mathrm{Ob}[\mathrm{is}] \mathrm{po}-$

16. $\mathrm{S}[\mathrm{eño} \mathrm{r} \mathrm{r} \mathrm{Ob}[\mathrm{is}]$ po aora te contare Como tu buen hijo que ya llego

17. tiempo que se hiso Convento en Guasamota yantraido 
18. un $\mathrm{p}[\mathrm{adr}] \mathrm{e}$ de Guadiana, $\mathrm{q}[\mathrm{u}] \mathrm{e}$ se llama fran $[\mathrm{cis}]^{\mathrm{co}}$ fila yen llegando a

19. Guasamota le di mis subditos q[u]e le aiudasen a hacer El

20. convento ysirben al Rei yala Casa de Dios y le doi ta

21. pisques $\mathrm{y}$ lo hago bien $\mathrm{y}$ los coras del mesmo modo $=\mathrm{y}$

22. Los de ayotuspa tambien tienen Convento, y mis subditos

23. Sirven al Rei y Los de Ayotuspa y Cora $=$

24. Y tambien q[uan]do se hizo El Convento de Guaxicori todos mis sub

25. ditos sirvieron al Rei en su Casa y di tapisques y esto quiero

26. que sepas $\mathrm{S}[\mathrm{eño} 0] \mathrm{r} \mathrm{Ob}[\mathrm{is}]$ po porque te huelgues, y con esto doi fin

27. a mi Rasonam $[i e n]{ }^{\text {to }}$ porque lo sepas yo D[on] fran $[\text { cis }]^{\text {co }}$ Nayari $=$

Otro

28. $\mathrm{S}[$ eño] $\mathrm{rb}[\mathrm{is}] \mathrm{po}$ ahora piden mis hijos que pues an trabajado no los

29. obliguen atributar sino que los dejen Sembrar y hacer

30. sus Casas, y p[ar]a eso den q[uen]ta al S[eño]r Ob[is]po=

31. Ya le Dije a D[on] felipe El g[obernad $]^{\text {or }}$, $q[u]$ e nos juntemos y nos ayu

Foja 2 recto

1. aiudemos, $\mathrm{q}[\mathrm{u}] \mathrm{e}$ asi se lo digo al S[eño]r Ob[is]po, y Con esto acabo

2. mi rasonam[ien] to $\mathrm{p}$ [ara] a que lo sepas y te huelgues y alegres yo lo

3. digo $\mathrm{q}[\mathrm{u}]$ e me llamo D[on] fran[cis ${ }^{\mathrm{co}}$ Nayari=

4. S[eño]r $\mathrm{Ob}[\text { is] po si nos quieres ya entiendes } n \text { [uest] ro Rasonam[ien }]^{\text {to }} \mathrm{y}$ que

5. remos que nos correspondas $=$

6. Y también n[uest]ro famoso y amado S[eño]r El Rei nos debe amar

7. Con todo su Corazón: Como nosotros Le amamos aunque esta

8. Lejos en españa deveras Le amamos Con todo n[uest] ro Corason

9. y Con esto Vasta este Rasonam[ien ${ }^{\text {to }} \mathrm{p}[\mathrm{ar}] \mathrm{a} \mathrm{q}[\mathrm{u}] \mathrm{e}$ lo entiendas ques bueno

10. Aora te digo otro Rasonam[ien $]^{\text {to }}$ que aquí vino Miguel

11. de la Cuara [Caldera], y me baptiso y puso por $\mathrm{n}$ [ombr]e D[on] fran[cis] ${ }^{\mathrm{c}}$ Nayari

12. y Otra ves vino Ba[rtolo] ${ }^{\text {me }}$ Xuarez El Cap [it $]^{\text {an }}$ y Baptiso mi hijo

13. y le puso por $\mathrm{n}$ [ombr]e $\mathrm{D}$ [on] Miguel geronimo y fue su padrino 
14. y lo supo El S[eño]r Ob[is]po, y Con esto acabo mi Rasonam[ien $]^{\text {to }}$ y fir

15. $\mathrm{mo}=\mathrm{D}[\mathrm{on}]$ fran $[\mathrm{cis}]^{\mathrm{co}}$ Nayari $=$

Foja 2 vuelta

1. Nayarit $=$

2. Año de 1649

3. Carta escrita por un Yndio Gover

4. nador al S[eñ]or Colmenero dandole

5. cuenta de su fidelidad al Rey, y su

6. amor a la Religion 
DOCUMENTO EN NÁHUATL DE LA CARTA DE FRANCISCO NAYARI, 1649

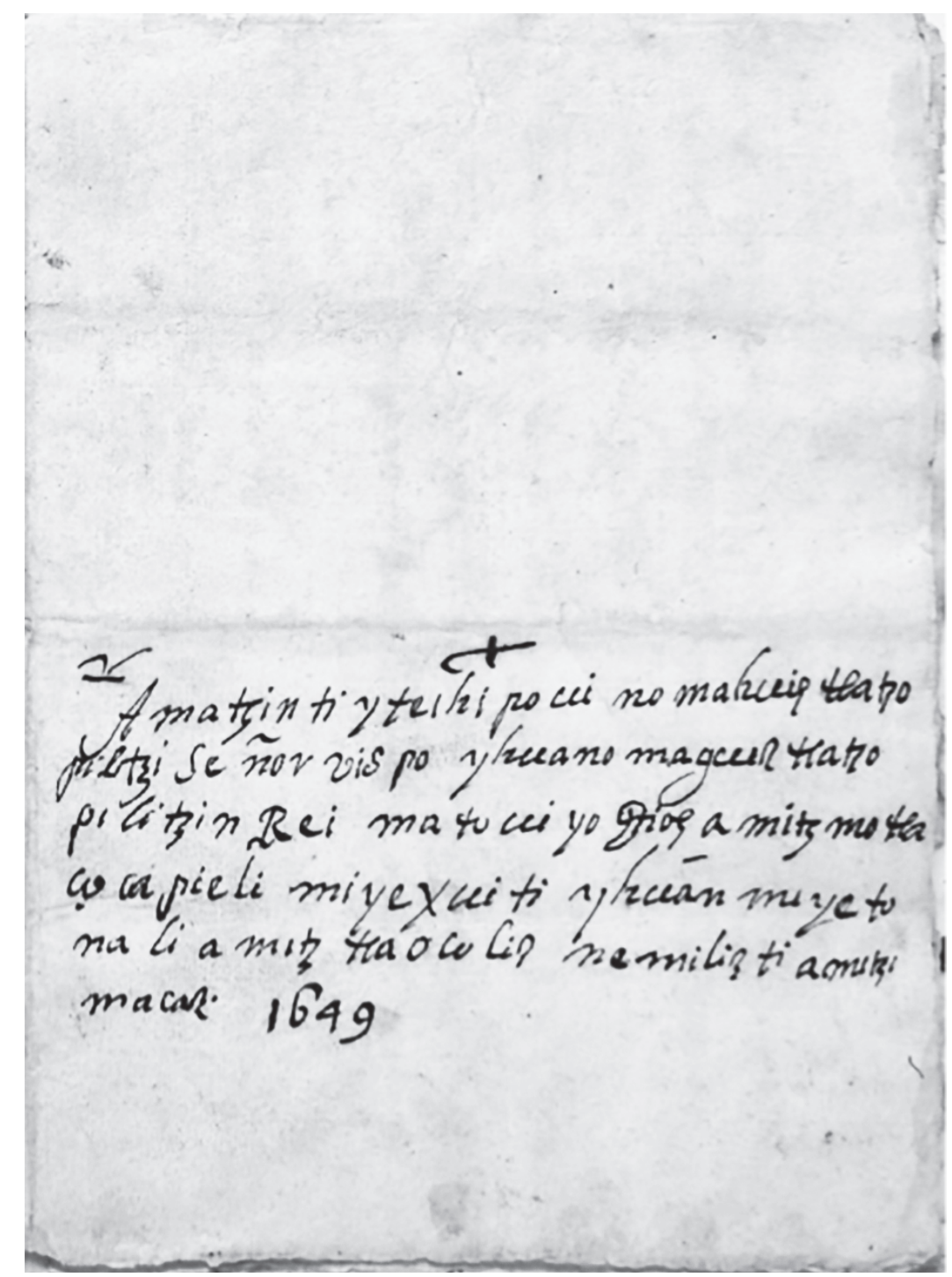

Imagen 1. Foja 1 recto 


\section{2

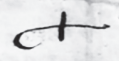

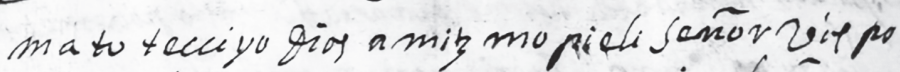

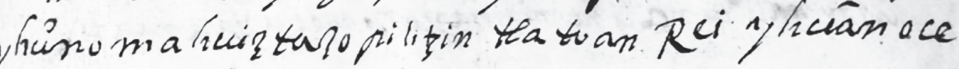

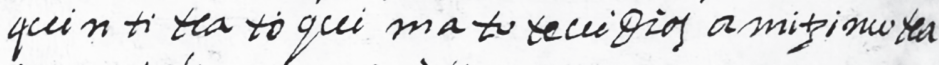
3. conpicli mirexiceiti

rizcein negcerti no tucabonfon manari te teccizy gior nichi mu ma qevilia mitrino

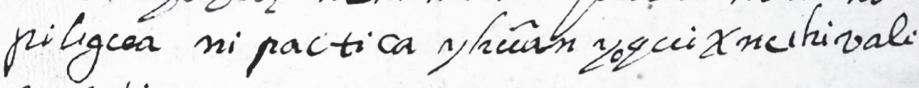
matica

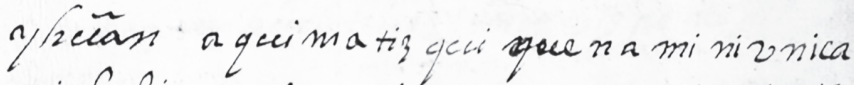
ni lfirtiano ni 20 ca quenami 2 nixtali

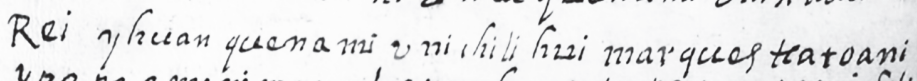
ypapa a nzo ni mo ne Los y ma fuacate pe uani vni filicis

tha toan marqueg axca nimatitica nivnica te vquituteatoa nilausa a a nuo ni moneloa san no yoquineifrico colia te peceani mila vacattavalilow a mo ni mo neloa yna vaca neguati nicura molhi no piligean quasamo tacorami ylician ayotultipa no piliguan curami -. 1/rceann yceax cure no piligua co ra mi ya quil hitimati? Señor vis pu ylucan Rci ef pania v nica $v$ mytra mo poal.

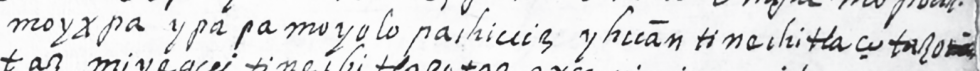

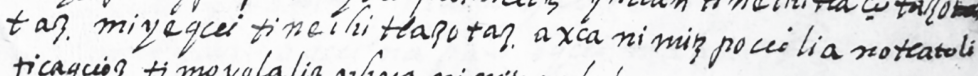
ticaquion timoyolalin a/rea ni misyo la lia a mo te no no teated a colea linivnica Señor vis polaceli nimistla tau tia tineshipalecuin nicquine quindvatili yehera tiv policit ma cay a marques $1 / p a p a$ mo pias amati pet to camo ta no ab te gece piaz amati nni nic qui ne quei 
1 1ruan nimptestanila no navatili If cas to li tur linemi may vomoliza anati

Imagen 3. Foja 2 vuelta 


\section{$\Upsilon$}

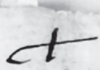

ma to tevi yogior nny mo puelis sernor rois po no ma ficur

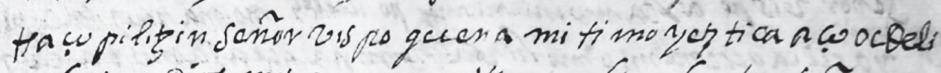

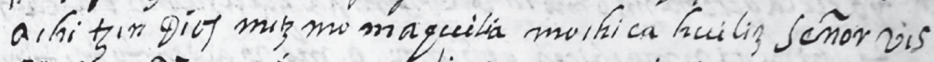
do amagiag minmo pecti mlyoxeciti

sicangricaqui mo ma haigteatotin mo te ta culilin mo tequi patroliz vnicaqui ruali cenca vni pa quei mimoyolali nitemamic moc $x$ bin 1 racean 2 momatin y raân mo cerona oni tenamic spa pa mo fecelitilir sênor vis po no pilizin (1)

aXa mulfi nitealiz upaamatin papacili temex tuqui parara no pilizin Señov vispo quecnami oni quil ricea yacenca

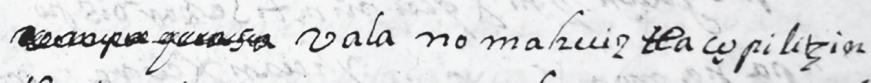
thaturni marques o a cirlia pe ta oquinocha niman veja om pao aci oqui na miege yextin

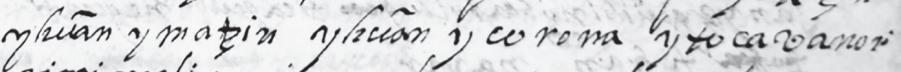
nayazing quali o quei ra pabo oycei ma huintiliyogceiromo

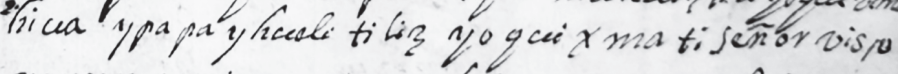

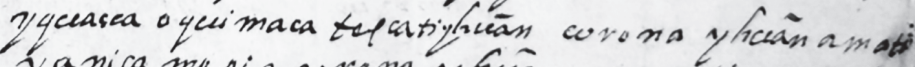
ta nica mu pia curoma 1 fricam a ma ti yaopolicu topcatinicavnia mopia R-cila Xca ymixmat. 
Señor wispo axca mu $\sqrt{2 i}$ nimy po lucibig no ma acein tacupilition yacuicayuenamio mo hizea cem pa qua samu ta cano terlicuparomorricea quobento a mo valicatutatin gecariana y te ca rieafica to jin o a ci gceasa muth netellic cupa 20 morlucua y racea no piliguean 20 ni

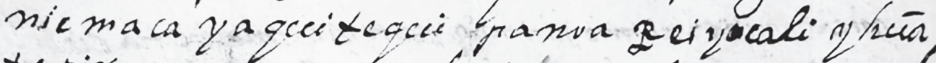

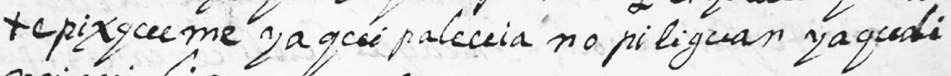
oniquilriza y/zeca curami

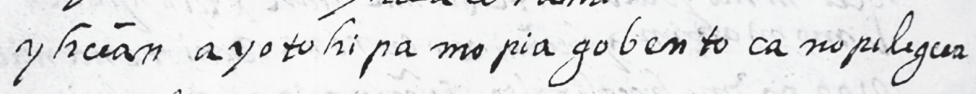

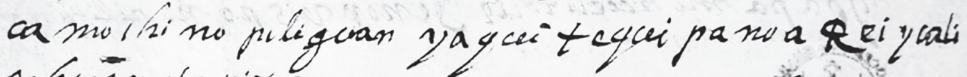
1) huan tepix qcee me anotutipacoramb

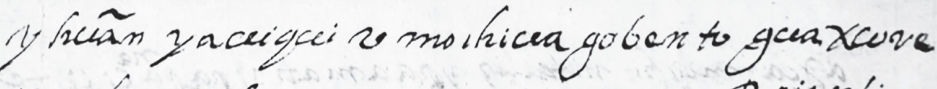
motrino giligea yaguite qui panoa Reizeali 2) frawn te frixacime yog ceci nite maca no pituguan zni $X$ acei matiseñorois po

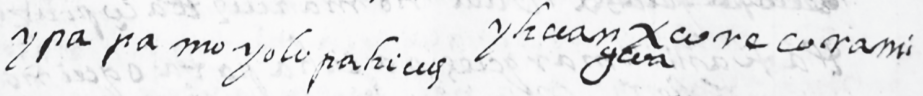
Janylgecirlinuteatuli ticagcuip neyceati som tujan nayar

Imagen 5. Foja 3 vuelta 
ieñorvispo aXcatic nequi no piligcean yacuiquetenti yainniclitetiqui tor a mo mextequipalsos ni trmacas tace teace mocarfuceas y pa pa no 20

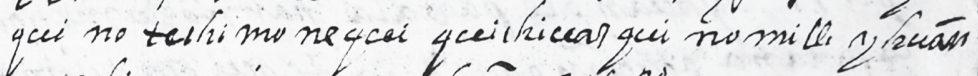
nocali rogcuix mat. Señor vis po

yaznigacelles oon fingo filibe

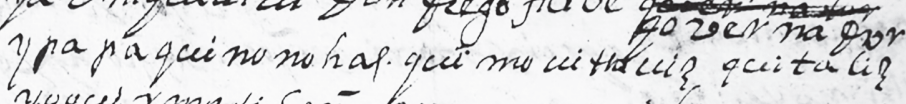

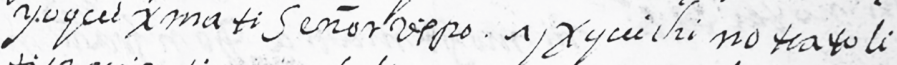
picaquin timo valiz timu ra pagccilltin nimor.

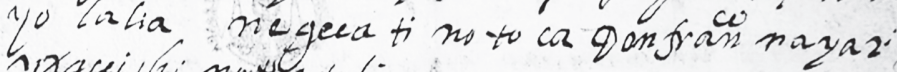
yaquilai novetati

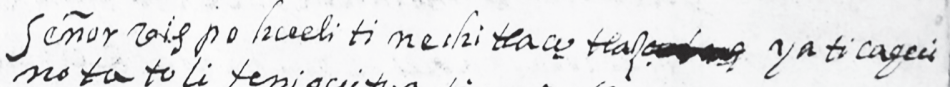
nota to li teniquitua tiner teace camatis

ylueian no makuin this Rei ma ne? tealutaca y camo, livo to queema

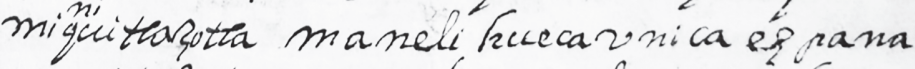

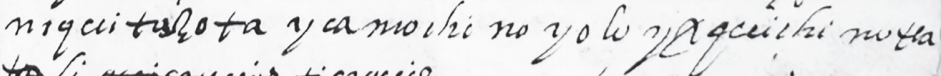

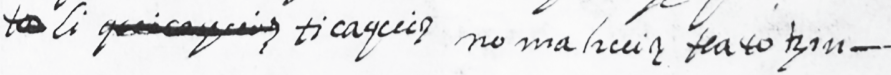


a) ca nimy matsilitiz no tha tuli nicavala

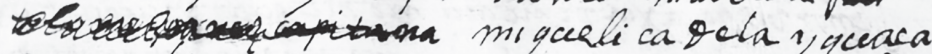
ay meiliqua tequi nol tu cayoti gun fir and na zari yruàn oce pavala partulurmeloaves apitana y rewans o qui qua tequi no pilutix oyceito can oti Bomi queli ceronimo 2/ teation

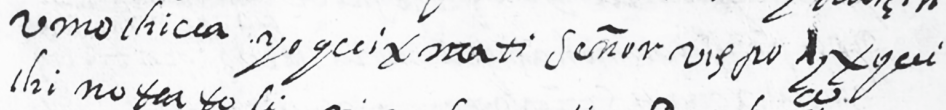
Whi noted to $C^{\circ}$ nimofimatia gom fram incinon

Imagen 7. Foja 4 vuelta 
DOCUMENTO DE LA TRADUCCIÓN AL ESPAÑOL DE LA CARTA DE FRANCISCO NAYARI 1649

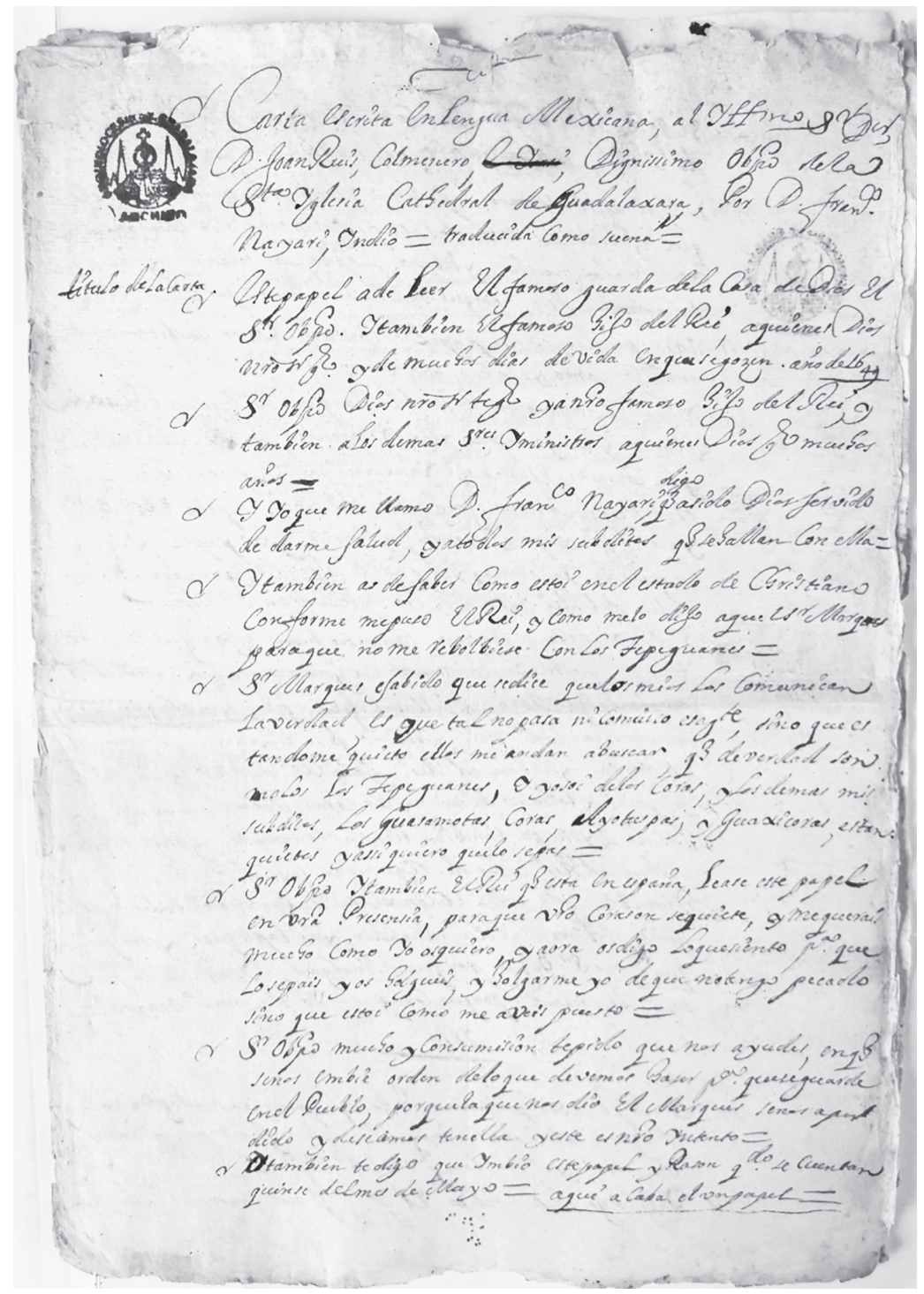

Imagen 8. Foja 1 recto 


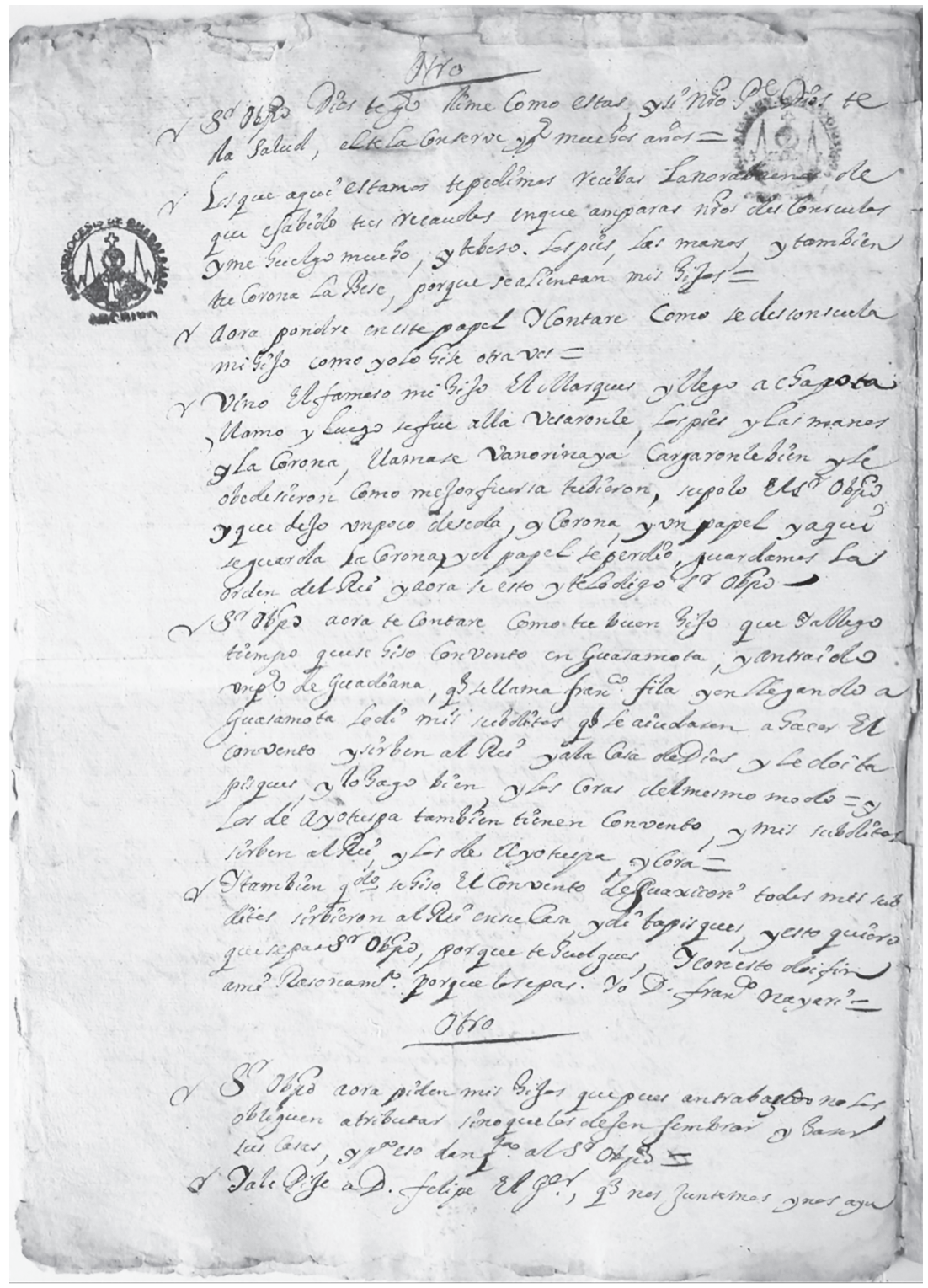

Imagen 9. Foja 1 vuelta 


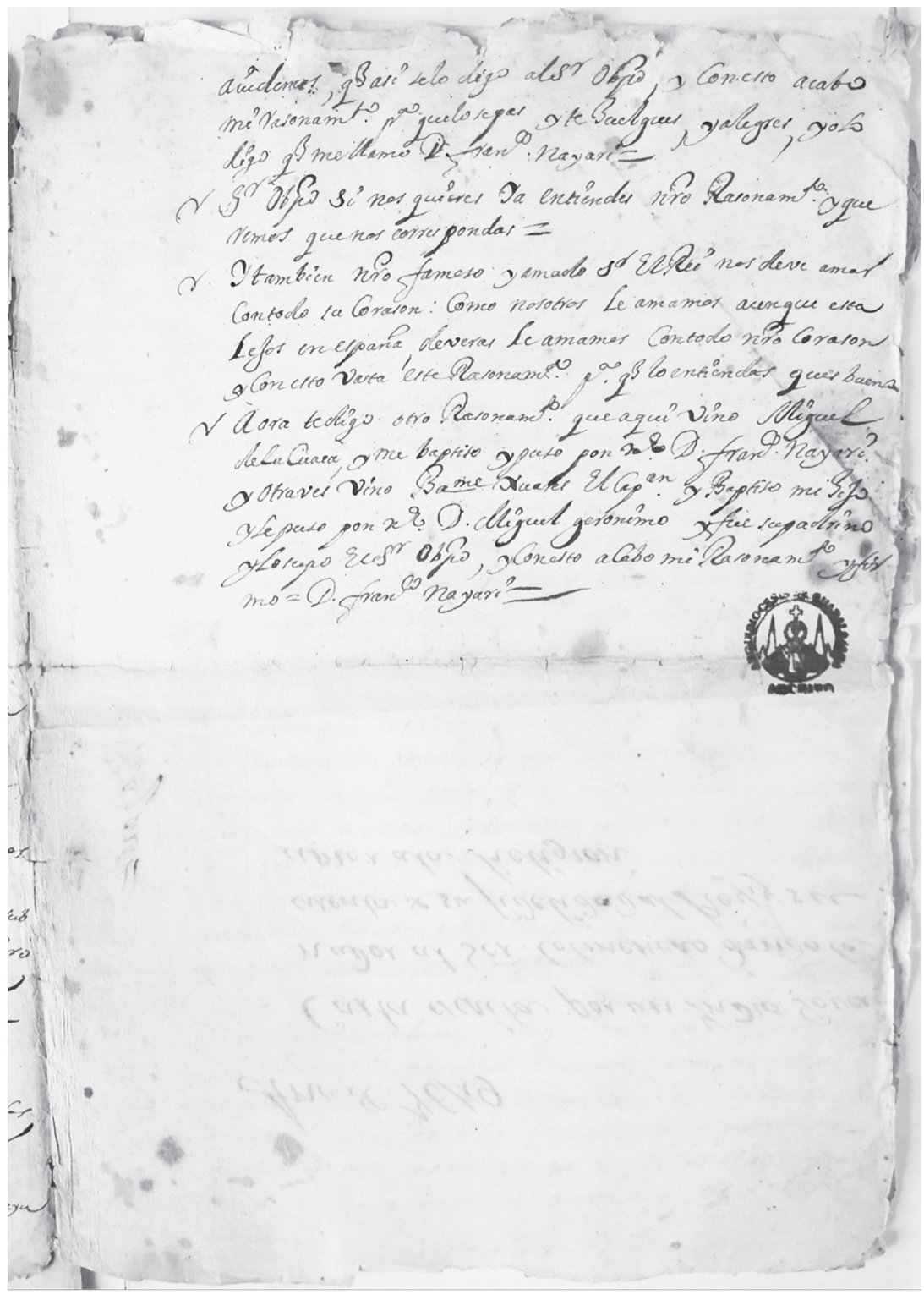

Imagen 10. Foja 2 recto 


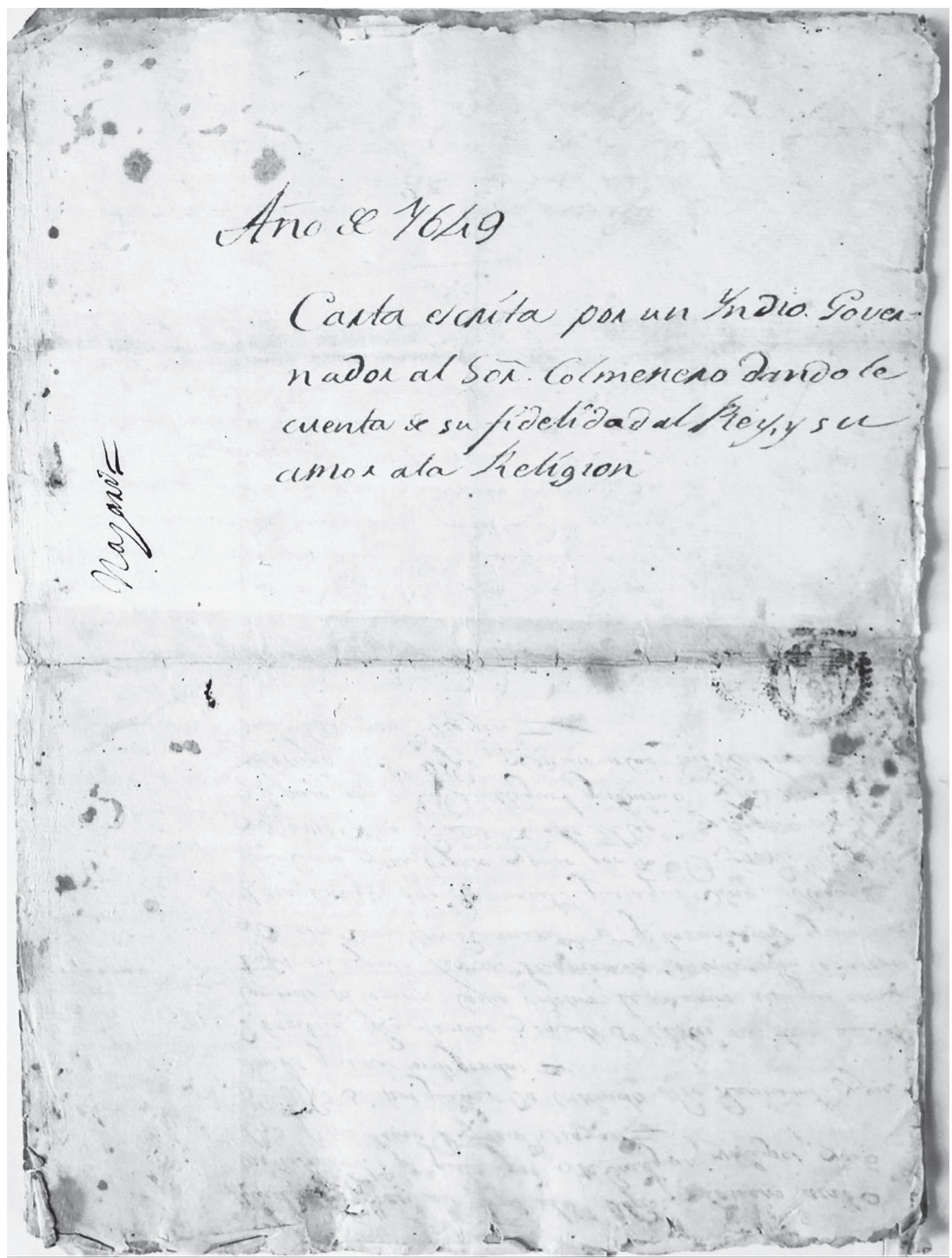

Imagen 11. Foja 2 vuelta 


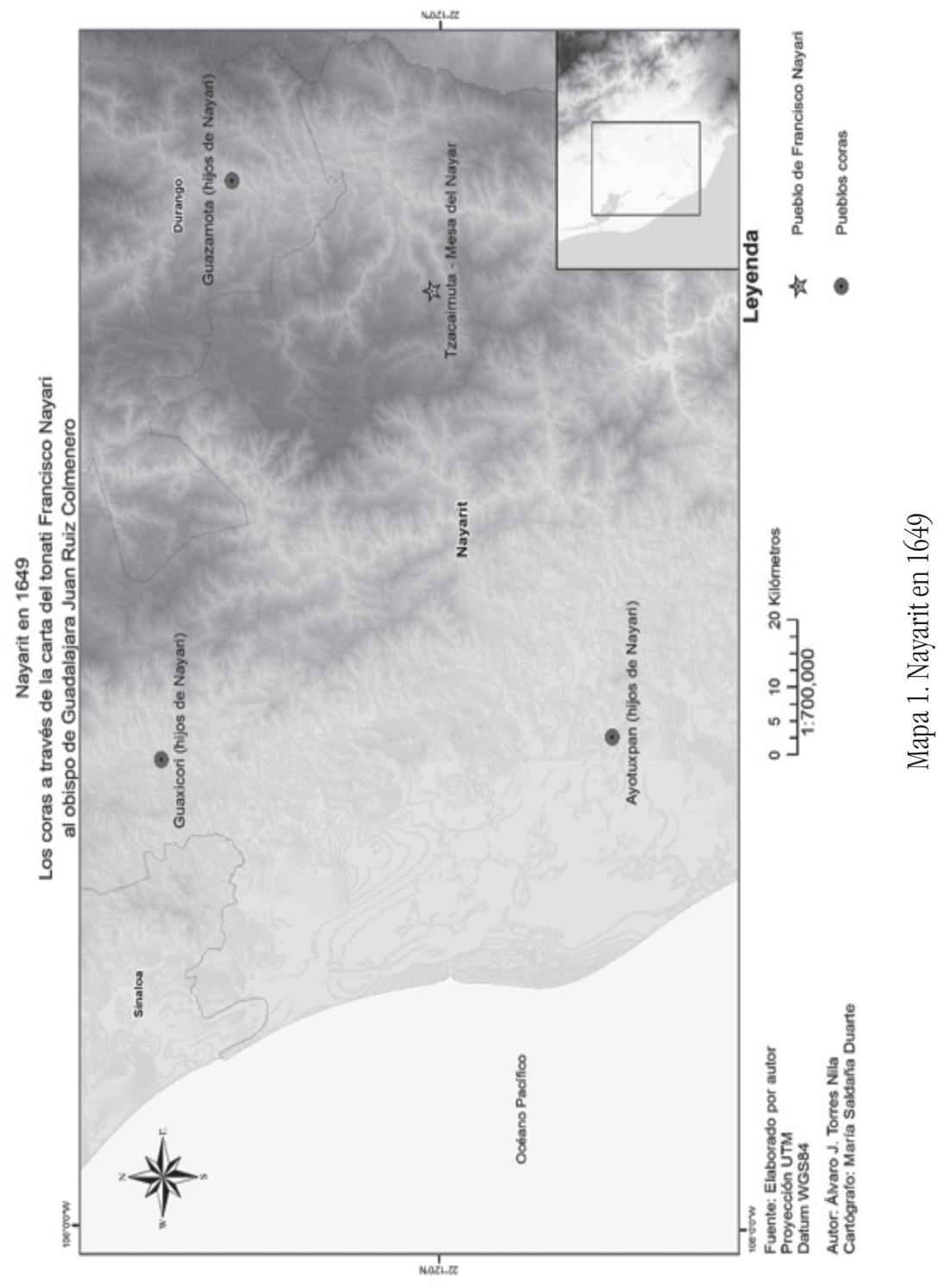




\section{Abreviaturas}

$\begin{array}{ll}1 & \text { primera persona } \\ 2 & \text { segunda persona } \\ 3 & \text { tercera persona } \\ \text { A } & \text { agentivo } \\ \text { ABS } & \text { absolutivo } \\ \text { ADV } & \text { adverbio } \\ \text { APL } & \text { aplicativo } \\ \text { AUX } & \text { auxiliar } \\ \text { CAUS } & \text { causativo } \\ \text { CONJ } & \text { conjunción } \\ \text { DEM } & \text { demostrativo } \\ \text { DIR } & \text { direccional } \\ \text { FUT } & \text { futuro } \\ \text { H } & \text { honorífico } \\ \text { IMP } & \text { imperativo } \\ \text { INSTR } & \text { instrumental } \\ \text { IPFV } & \text { imperfecto } \\ \text { LOC } & \text { locativo } \\ \text { OBJ } & \text { objeto } \\ \text { PERF } & \text { perfecto } \\ \text { PL } & \text { plural } \\ \text { POS } & \text { posesivo } \\ \text { POSP } & \text { posposición } \\ \text { PRET } & \text { pretérito } \\ \text { PROG } & \text { progresivo } \\ \text { REFL } & \text { reflexivo } \\ \text { SG } & \text { singular } \\ \text { SUJ } & \text { sujeto } \\ & \end{array}$

\section{Referencias}

Archivo consultado

ARCHIvo Histórico de la Arquidiócesis de Guadalajara (AHAG). Documentos en Náhuatl.

Obras y Estudios

CORTÉS Y ZEDEÑNo, Gerónimo Thomas de Aquino

1765 Arte, Vocabulario y Confessionario en el Idioma Mexicano, Como se usa en el 
Obispado de Guadalaxara. Puebla: Imprenta del Colegio Real de San Ignacio de la Puebla de los Ángeles.

DÁvILA GARIBI, José Ignacio

1957 Apuntes para la Historia de la Iglesia en Guadalajara, tomo I. México: Editorial Cvltura.

DELARRosa, Francisco

1997 "Entrada del obispo de Guadalajara en la provincia de los coras nayaritas los días 5 y 6 de marzo de 1649", Relaciones. Estudios de Historia y Sociedad 69: $79-83$.

DuRÁn, fray Diego

1880 Historia de las Indias de Nueva España y Islas de Tierra Firme, tomo II, México: Imprenta de Ignacio Escalante.

GüERECA DuRÁn, Raquel Eréndira

2018 “Caciques, 'Lenguas’ y Soldados Fronterizos: Actores Indígenas en la Conquista del Nayar, 1721-1722”. Tesis doctoral. México: Universidad Nacional Autónoma de México.

HERrera, Carmen, Valentín PERALTA, Brígida von MENTZ y Elsie RoCKwEll

2007 "La repentina muerte de Catalina Toztlapal: análisis y traducción de un texto en náhuatl de 1562”, en Estructura, Discurso e Historia de Algunas Lenguas Yutoaztecas, Ignacio Guzmán Betancourt, José Luis Moctezuma Zamarrón, coordinadores, pp. 149-184. México: Instituto Nacional de Antropología e Historia.

HERS, Marie-Arieti

1977 "Los coras en la época de la expulsión jesuita", Historia Mexicana XXVII (1), julio-septiembre: 17-48.

MEDINA GARCíA, Ricardo

2016 "Nahuatl-Language Petitions and Letters from Northwestern New Spain, 15801694". Tesis doctoral. University of California, Los Angeles.

MENTZ, Brígida von

2009 “Cambio social y cambio lingüístico, el 'náhuatl cotidiano', el de 'doctrina', y el de 'escribanía' en Cuauhnáhuac entre 1540 y 1671”, en Visiones del Encuentro de Dos Mundos en América; Lengua, Cultura, Traducción y Transculturación, Karen Dakin, Mercedes Montes de Oca y Claudia Parodi, editoras, pp. 111145. México: Universidad Nacional Autónoma de México.

MoLina, fray Alonso de

1571 Vocabulario en Lengua Mexicana y Castellana. México: Casa de Antonio Spindola. 
NEURATH, Johannes

2002 Las Fiestas de la Casa Grande. México: Instituto Nacional de Antropología e Historia / Universidad de Guadalajara.

ORTEGA, José de

1996 "Libro I. Maravillosa reducción y conquista de la Provincia de San Joseph de Gran Nayar, nuevo Reino de Toledo", en Apostólicos Afanes de la Compañia de Jesús en su Provincia de México, Francisco Javier Fluviá, editor, pp. 1-223. México: Edición Facsimilar, Centro Francés de Estudios Mexicanos y Centroamericanos / Instituto Nacional Indigenista.

Peralta, Valentín, Carmen Herrera, Constantino Medina, Brígida von Mentz, Elsie Rockwell y Zazil SANDOVAL

2004 "Traducción de documentos en náhuatl: una perspectiva interdisciplinaria”, Estudios de Cultura Nábuatl 35: 179-206.

PERón, Mylène

1997 "Dos visitas episcopales del siglo XVII en la sierra de Nayarit. Intereses en juego y límites de la conquista espiritual del occidente mexicano", Relaciones. Estudios de Historia y Sociedad 69: 42-76.

POWELL, Philip W.

1977 La Guerra Chichimeca (1550-1600). México: Fondo de Cultura Económica. RANGEL GuZMán, Efraín y Jorge Luis Marín GARCía

2014 "Desplazamientos territoriales y nuevos asentamientos tepehuanes", Relaciones. Estudios de Historia y Sociedad 35 (137): 149-178.

ROJAS RABIELA, Teresa

1979 "La organización del trabajo para las obras públicas: el coatequitl y las cuadrillas de trabajadores", en El Trabajo y los Trabajadores en la Historia de México, Elsa Cecilia Frost, Michel C. Meyer y Josefina Zoraida Vázquez, compiladores, pp. 41-66. México: El Colegio de México / University of Arizona Press.

SanToscoy, Alberto

1899 Nayarit. Colección de Documentos Inéditos, Históricos y Etnográficos acerca de la Sierra de este Nombre. Guadalajara: Tipo-Lit. y Enc. de José María Yguiniz. TÉllez Lozano, Víctor Manuel

2014 "Acercamiento al estudio de los sistemas de cargos entre las comunidades huicholas de Jalisco y Nayarit, México", Diálogo Andino 43: 17-40.

VázQueZ SoTo, Verónica

1994 "Los conceptos de propiedad en cora: modificación, predicación y marcación de número" en Investigaciones Lingüísticas en Mesoamérica, Carolyn J. MacKay y 
Verónica Vázquez, editoras, pp. 147-181. México: Universidad Nacional Autónoma de México.

YáÑez Rosales, Rosa H.

2001 Rostro, Palabra y Memoria Indígenas. El Occidente de México: 1524-1816. México: Centro de Investigaciones de Estudios Superiores en Antropología Social, Instituto Nacional Indigenista (Colección Historia de los pueblos indígenas de México).

2008 "La evangelización en náhuatl en la época colonial: documentos de la Audiencia de Guadalajara”, Tlalocan XV: 145-162.

2017 "Nahuatl L2 texts from Northern Nueva Galicia. Indigenous language contact in the seventeenth century", en Language Contact and Change in Mesoamerica and Beyond, Karen Dakin, Claudia Parodi y Natalie Opertein, editoras, pp. 237-261, volumen 185. Amsterdan - Phliladelphia: John Benjamins Publishing Company.

Recibido: 17 de mayo de 2019

Aceptado: 6 de octubre de 2019 
